\title{
Natural hydroxyanthraquinoid pigments as potent food grade colorants: an overview
}

\author{
Yanis Caro, ${ }^{\mathrm{a}, \mathrm{b}, *}$ Linda Anamale, ${ }^{\mathrm{a}, \mathrm{b}}$ Mireille Fouillaud, ${ }^{\mathrm{a}, \mathrm{b}}$ Philippe Laurent, ${ }^{\mathrm{b}, \mathrm{c}}$ Thomas Petit, $^{\mathrm{b}, \mathrm{c}}$ and \\ Laurent DuFOSSE ${ }^{\mathrm{a}, \mathrm{b}}$ \\ ${ }^{a}$ Département Agroalimentaire, ESIROI, Université de La Réunion, Sainte-Clotilde, Ile de la Réunion, France \\ bCSNSA, Faculté des Sciences et des Technologies, Université de La Réunion, Sainte-Clotilde, Ile de la Réunion, France \\ ${ }^{c}$ Département Génie Biologique, IUT, Université de La Réunion, Saint-Pierre, Ile de la Réunion, France
}

Received 24 October 2012; Accepted 12 November 2012

(C) The Author(s) 2012. This article is published with open access at Springerlink.com

\begin{abstract}
Natural pigments and colorants are widely used in the world in many industries such as textile dying, food processing or cosmetic manufacturing. Among the natural products of interest are various compounds belonging to carotenoids, anthocyanins, chlorophylls, melanins, betalains... The review emphasizes pigments with anthraquinoid skeleton and gives an overview on hydroxyanthraquinoids described in Nature, the first one ever published. Trends in consumption, production and regulation of natural food grade colorants are given, in the current global market. The second part focuses on the description of the chemical structures of the main anthraquinoid colouring compounds, their properties and their biosynthetic pathways. Main natural sources of such pigments are summarized, followed by discussion about toxicity and carcinogenicity observed in some cases. As a conclusion, current industrial applications of natural hydroxyanthraquinoids are described with two examples, carminic acid from an insect and Arpink red $^{\mathrm{TM}}$ from a filamentous fungus.
\end{abstract}

Keywords: anthraquinone, hydroxyanthraquinone, natural colorant, food colorant, microbial pigment, biotechnology, mycotoxin contamination

\section{Introduction}

Food grade colorants can loosely be categorized as 'natural' or 'synthetic'. The term 'natural colorants' indicates that the source of the colorant is natural even if varying definitions and regulations exist according to the country in question. For example, under the United States (US) Food and Drug Administration (FDA) regulations, a colorant added to a food product cannot be considered as 'natural', no matter what the source is; unless the colorant is natural to the food product itself. The FDA regulates the natural and synthetic colorants of food applications in two classes. In general, the synthetic colorants (that do not exist in nature) are subjected to a certification requirement to assure that each batch of material manufactured meets the standard specifications, while natural colorants are "exempt from certification" and may be manufactured and marketed without certification of FDA (no US Food, Drug and Cosmetic Act (FD\&C)-number). In contrast, E-numbers are used for all colorants for food applications in the European Union (EU). Colorants for food applications listed by both the FDA and the EU are tested for biosafety before their promotion and commercialization, and are further controlled by national legislation specifying those

*To whom correspondence should be addressed. E-mail: yanis.caro@univ-reunion.fr colorants that may be used, the type of food that may be coloured, the quantity that may be added and the limit of maximum daily intake.

For a very long time, the use of food colorants focused on synthetic ones. However, over the last few decades, synthetic colorants tend to be perceived as undesirable by consumers, due to the harmful effects of some synthetic pigments on human health, including allergic reactions, mutagenicity and potential carcinogenicity (e.g. skin cancer) ${ }^{1}$. Many manufacturers have considered replacing synthetic colorants in their food products with natural colouring alternatives in response to pressure from both customers and regulators ${ }^{2}$. Whereas 43 colorants were authorized in the EU as food additives in 1994, actually almost a hundred of food grade colorants are authorized in the EU and have been assigned by an 'E-number'; almost $40 \%$ of these were of natural origin ${ }^{1,2}$. These natural colorants are usually applied in several industrial food processes for the same reasons as the synthetic counterparts: (i) to enhance the product's natural colour whose ingredients are unable to provide a sufficient colour; (ii) to standardize the colour and appearance of product like confectionery; (iii) to restore what has been lost during processing or (iv) to add a novel sensory aspect that attracts customers.

Among the natural pigments of interest are various compounds belonging to carotenoids, anthocyanins, 
chlorophylls, melanins, betalains, quinones... This review emphasizes pigments with anthraquinoid skeleton and gives an overview on hydroxyanthraquinoid pigments which are widely present in Nature and are gaining increasing interest by academics and people from the industry.

\section{Trends in consumption, production and regulation of} natural food grade colorants in the current global market

Currently, the natural food colouring industry market is growing $10 \%-15 \%$ annually. Natural varieties share of the global food colorant market increased from about $31 \%$ in 2005 to $36 \%$ in 2009 . The current consumer preference for natural food grade colorants is associated with their image of being healthy and of good quality. According to a report from Leatherhead Food Research, Shaun Weston mentions that the global market for food grade colorants is expected to reach $\$ 1.6$ billion USD by 2015 , up to $10 \%$ from its present levels and fuelled mainly by the growth in natural colorants and colouring foodstuffs (data from Leatherhead Food International LFI) (www.leatherheadfood.com). The main industrial technology used for the production of natural colorants for food applications depends on the extraction of coloured pigments from edible plants, fruits or vegetables. Table 1 shows the main natural food grade colorants authorized and currently available in the current global market. Common natural colorants include turmeric, curcumin, annatto, paprika, caramel and cochineal extract. Natural colorants are often commercially available in powder, oil-soluble emulsion, or water-soluble emulsion forms.

Table 1. Main natural food grade colorants authorized and currently available in the current global market

\begin{tabular}{|c|c|c|c|}
\hline Color/shade & E-number* & \multirow[t]{2}{*}{ Natural colorant } & Chemical category \\
\hline \multicolumn{3}{|c|}{ From plants, fruit or vegetables: } & \\
\hline Yellow & E100, E100 (i) & Curcumin & Curcuminoid \\
\hline Yellow & E100 (ii) & Turmeric & Curcuminoid \\
\hline Green & E140 & Chlorophylls & Tetrapyrrole \\
\hline Green & E141 & Chlorophyllins & Tetrapyrrole \\
\hline Brown & E150a-d & Caramel & Melanoidin \\
\hline Orange-yellow & E160a (i) & Mixed Carotenes & Carotenoid \\
\hline Orange-yellow & E160a (ii) & $\beta$-carotene & Carotenoid \\
\hline Yellow to orange & $\mathrm{E} 160 \mathrm{~b}$ & Annatto & Carotenoid \\
\hline Yellow to orange & E160b (i) & Annato (Bixin) & Carotenoid \\
\hline Yellow to orange & E160b (ii) & Annato (Norbixin) & Carotenoid \\
\hline Red & E160c & Paprika (Capsanthin) & Carotenoid \\
\hline Yellow to red & E160d & Lycopene & Carotenoid \\
\hline Yellow to red & E160e & Apocarotenal & Carotenoid \\
\hline Orange-yellow & E161a & Flavoxanthin & Carotenoid \\
\hline Orange-yellow & E161b & Lutein & Carotenoid \\
\hline Orange-yellow & E161d & Rubixanthin & Carotenoid \\
\hline Orange-yellow & E161e & Violaxanthin & Carotenoid \\
\hline Orange-yellow & E161f & Rhodoxanthin & Carotenoid \\
\hline Orange, Red & E161h & Zeaxanthin & Carotenoid \\
\hline Red & E162 & Red Beet Juice & Betalain \\
\hline Red, Blue or Violet & E163a & Cyanidin & Anthocyanin \\
\hline Red, Blue or Violet & $\mathrm{E} 163 \mathrm{e}$ & Peonidin & Anthocyanin \\
\hline
\end{tabular}

\begin{tabular}{llll}
\hline From animal: & E101, E101a & $\begin{array}{l}\text { Riboflavin } \\
\text { Carminic acid } \\
\text { (Cochineal extract) }\end{array}$ & Flavin \\
Magenta-red & E120 (ii) & $\begin{array}{l}\text { Cryptoxanthin } \\
\text { Canthaxanthin }\end{array}$ & Carotenoid \\
Carotenoid
\end{tabular}

From microorganisms:

\begin{tabular}{|c|c|c|}
\hline Yellow & E101 (iii) & $\begin{array}{l}\begin{array}{l}\text { Riboflavin } \\
\text { (from Bacillus subtilis) }\end{array} \text { Flavin } \\
\text { Other sources: Ashbya gossypii, } \\
\text { Candida gulliermndii, acetobutylicum } \\
\text { Clostridium a } \\
\text { and Debaryomyces subglbosus }\end{array}$ \\
\hline Orange-yellow & E160a (ii) & $\begin{array}{l}\boldsymbol{\beta} \text {-carotene Carotenoid } \\
\text { (from Blakeslea trispora) }\end{array}$ \\
\hline Orange-yellow & E160a (iv) & $\begin{array}{l}\boldsymbol{\beta} \text {-carotene } \\
\text { (from Dunaliella salina) } \\
\text { Other sources: Dunaliella bardawil }\end{array}$ \\
\hline Yellow to red & E160d (iii) & $\begin{array}{l}\text { Lycopene Carotenoid } \\
\text { (from Blakeslea trispora) }\end{array}$ \\
\hline Yellow to red & E-161j & $\begin{array}{l}\text { Astaxanthin Carotenoid } \\
\text { (from Haematococcus pluvialis) } \\
\text { Other sources: Haematococcus } \\
\text { lacustris, Xanthophyllomyces } \\
\text { dendrorhous }\end{array}$ \\
\hline Orange, Red & E161g & $\begin{array}{l}\text { Canthaxanthin Carotenoid } \\
\text { (from Haematococcus lacustris) }\end{array}$ \\
\hline & & Other sources: Bradyrhizobium sp. \\
\hline
\end{tabular}

Many scientific papers describe the extraction, characterization and properties of natural pigments from fruits, vegetables, lichens and marine life ${ }^{1,3}$. However, the potential of these renewable resources as sources for new commercial natural food grade colorants would still be limited both by the manufacturing costs and the availability of the raw material, which would need to be cultivated in sufficient quantities for industrial extraction. The microbial pigment production by biotechnology would have the advantage of producing higher yields. This kind of pigment production is not at all dependent on the availability and external supply of particular raw materials. In addition, microbial pigments are often more stable and water-soluble than those of plant sources ${ }^{4,5}$. The really first European success story in pigment production using a microorganism is $\beta$-carotene (additive E-160a(ii); orange-yellow pigment) from the fungus Blakeslea trispora by $\mathrm{DSM}^{\mathrm{TM}}$. Among microalgae, some successful stories yield to efficient production of carotenoids using Dunaliella salina (e.g., $\beta$-carotene, additive E-160a(iv)) or Haematococcus pluvialis (e.g., astaxanthin, additive E-161j; yellow to red pigment) ${ }^{6}$ (Table 1). Nowadays, fermentative productions of natural food grade colorants are available in the global market ${ }^{7}$. This approval of microbial carotenoids as food colorants has strengthened the prospects for new natural colorants ${ }^{2}$.

However, some new microbial pigments might not be accepted if they were to be introduced into industrial food manufacturing today ${ }^{8}$. The commercially available Monascus pigments are a perfect example. These fungal pigments are natural azaphilone pigment mixtures. The red colorant obtained is produced commercially using strains of Monascus fungi in the Orient for centuries used as a food colorant for making red rice wine, red soybean cheese, meat and marine

\section{曾 Springer}


products. However, Monascus pigments are still not allowed as a food additive in either the US or the EU; there have been controversial views presented over their safe use ${ }^{8}$. This could be essentially because of the presence of the mycotoxin citrinin (yellow compound) and some other potential toxic metabolites which may occur in some batches with Monascus fungi ${ }^{9-11}$. The production of citrinin limits the commercial use of Monascus fungi as producers of natural food grade colorants ${ }^{12}$. Research continues on new azaphilone pigments produced from non-mycotoxigenic fungal strains, such as Epicoccum nigrum, Penicillium aculeatum or $P$. pinophilum - that are incapable of co-producing citrinin - in the prospects for new natural food grade colorants ${ }^{8,13}$. The case of the fungal Arpink red $^{\mathrm{TM}}$ colorant, i.e. a natural food colorant manufactured by the Czech company, Ascolor Biotech s.r.o. is also atypical. This company has produced a chomophore of the anthraquinoid type as a natural food colorant, by fermentation and bioprocess engineering using the strain Penicillium oxalicum var. Armeniaca CCM 8242 obtained from soil (the variety was never formally described). The Arpink red $^{\mathrm{TM}}$ colorant has received a two-year temporary approval by the EU for distribution as a food additive, exclusively in the Czech Republic from 2004 to 2006. The extraction, isolation and characterization of natural anthraquinoid pigments have been also reported from other filamentous fungi with different shades such as red, reddish brown, bronze and maroon.

\section{Natural hydroxyanthraquinoid pigments: chemical structures of the main colouring components, their properties and their biosynthetic pathways}

Anthraquinones are a class of compounds of the quinone family that consists of several hundreds of compounds that differ in the nature and positions of substituent groups. Anthraquinoid derivatives are derivatives of the basic structure 9,10-anthracenedione or also called 9,10-dioxoanthracene, i.e. a tricyclic aromatic organic compound with formula $\mathrm{C}_{14} \mathrm{H}_{8} \mathrm{O}_{2}$ and whose ketone groups are on the central ring in position C-9 and C-10. Figure 1 shows the skeleton structure of anthraquinoid derivatives. In general, for each anthraquinoid derivative there are eight possible hydrogens that can be substituted. The term 'hydroxyanthraquinoid (HAQN)' derivatives usually refers to derivatives of 9,10-hydroxyanthraquinone, i.e. derivatives of 9,10-anthraquinone where any number $n$ of hydrogen atoms have been replaced by $n$ hydroxyl (-OH) groups. In this case the number $n$ of hydroxyl group is indicated by a multiplier prefix (mono-, di-, tri-, up to octa-). The HAQN derivatives absorb visible light and are coloured, whereas strictly 9,10-anthraquinone derivatives are colorless like tectoquinone ${ }^{14}$.

Most HAQN colour compounds of natural origin have<smiles>[R16]c1c([R1])c([R4])c2c(c1[R4])C(=O)c1c([R5])c([R6])c([R8])c([R5])c1C2=O</smiles>

Figure 1. The skeleton structure of anthraquinoid derivatives complex structures with several functional groups, which modify their absorption spectra. The chemical structures of natural HAQN pigments and their main physical and functional properties ${ }^{15-52}$ are shown on Table 2. In the UV region, substituted 9,10-anthraquinone derivatives show intense benzenoid absorption bands fairly regularly within the ranges $240-260$ and $320-330 \mathrm{~nm}$. The quinonoid bands appear in a range from 260 to $290 \mathrm{~nm}$ and 9,10-hydroxyanthraquinone derivatives show an absorption band between 220 and $240 \mathrm{~nm}$. The HAQN derivatives have attracted the attention of many researchers due to their large list of possible applications related to their interesting photoactivity and more particularly based on their chromatic properties. They possess good light-fastness properties, which makes metallization unnecessary. HAQN derivatives can form coordination complexes with several cations. They are relatively stable and the advantage of pigments of HAQN-type compared to azo pigments is their superior brightness. Moreover, ionization of a hydroxylic group results in a bathochromic shift. It appears that the colour of the HAQN pigments depends on the position and number of the hydroxyl substituents in the different rings ${ }^{53}$.

Natural HAQN pigments are produced by the secondary metabolism of organisms. One of the remarkable features of natural HAQN biosynthesis is that they are derived from a variety of different precursors and pathways. There are at least two biosynthetic pathways leading to HAQN pigments. On one hand, the most important is the polyketide pathway (acetate-malonate pathway) that includes suitable folding and condensation of an octaketide chain derived from acetate (acetyl-CoA) and malonate (malonyl-CoA) units ${ }^{3,32,54}$. The resultant polycarbonyl compounds serve as substrates for various cyclases that produce aromatic compounds that represent typical fungal metabolites. Natural HAQN pigments that are synthesized following this acetate-malonate pathway (see Figure 2) always show a characteristic substitution pattern, i.e. they show substitution on both aromatic rings and more particularly at least one hydroxyl group in position R1 and one hydroxyl or methoxyl $\left(-\mathrm{OCH}_{3}\right)$ group in position $\mathrm{R} 8$ : examples being emodin, physcion, endocrocin, dermolutein, dermoglaucin, dermorubin and dermocybin. According to this polyketide pathway, the biosynthetic relationships show that the yellow compounds (e.g., emodin, physcion, endocrocin and dermolutein) exist in the beginning of the synthesis pathway whereas the red compounds like dermorubin and dermocybin are more complicated in structure and occur in the latter part of the biosynthesis pathway ${ }^{32}$. More recently, Bringmann et al. ${ }^{55}$ revealed that the pigment chrysophanol is shown to be formed, in an organism-specific way, by a third folding mode involving a remarkable cyclization of a bicyclic diketo precursor, thus establishing the first example of multiple convergence in polyketide biosynthesis.

On the other hand, HAQN pigments are formed via the shikimate or chorismate/o-succinylbenzoic acid pathway (Fig. 2). HAQN pigments that are synthesized via this pathway only have one of the rings unsubstituted and at least one hydroxyl group in position $\mathrm{R} 1$ on the ring $\mathrm{C}$. The rings $\mathrm{A}$ and $\mathrm{B}$ are derived from chorismate and $\alpha$-ketoglutarate via $o$ succinylbenzoic acid (the HAQN biosynthesis branch at 1,4dihydroxy-2-naphtoic acid), whereas ring $\mathrm{C}$ is formed from isopentenyl diphosphate either formed via the mevalonic acid pathway or the 2-C-methyl-D-erythritol 4-phosphate pathway ${ }^{54,56,57}$. The relevant colouring compounds are alizarin 
Table 2. Chemical structures, physical and functional properties of natural hydroxyanthraquinoid (HAQN) pigments

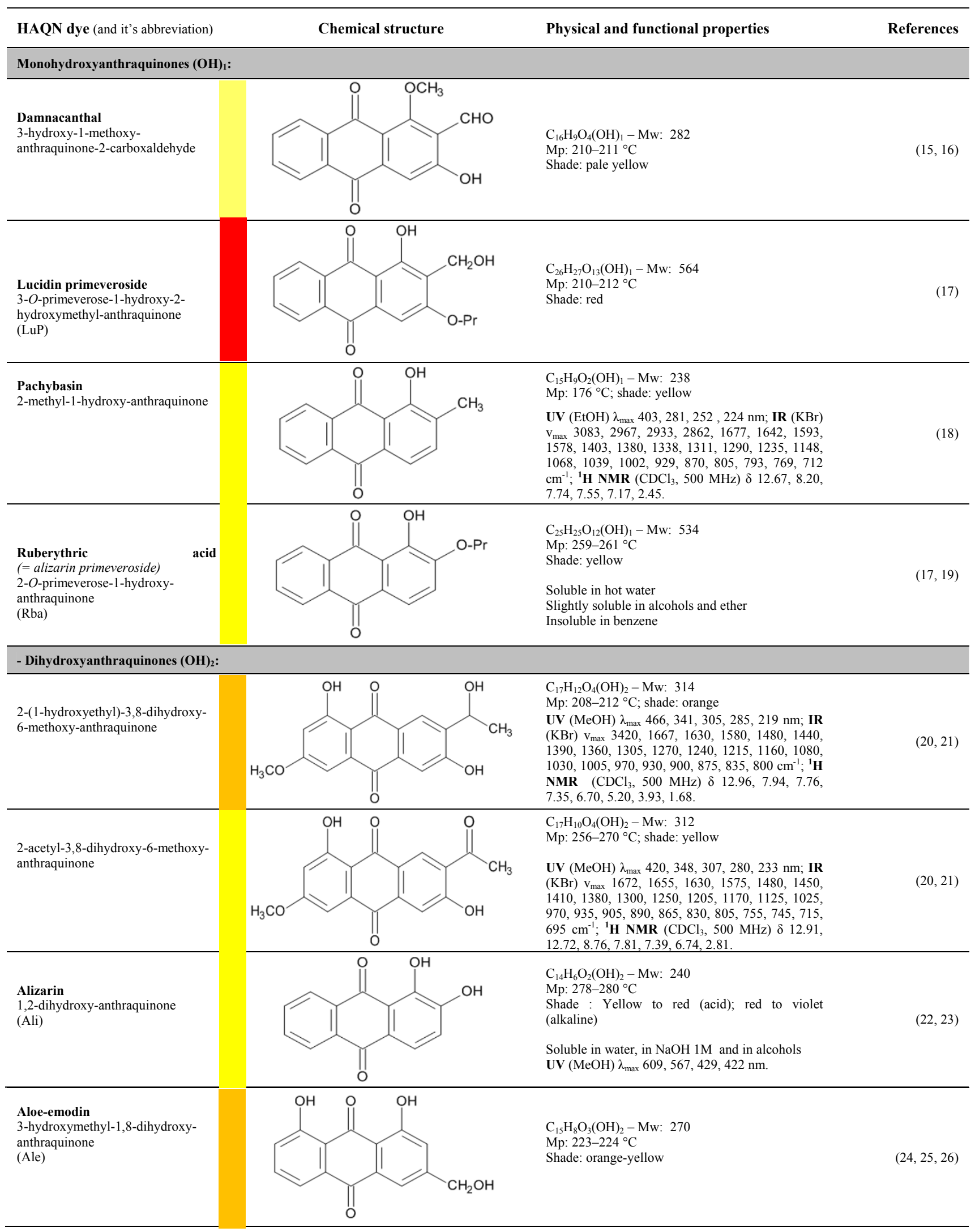




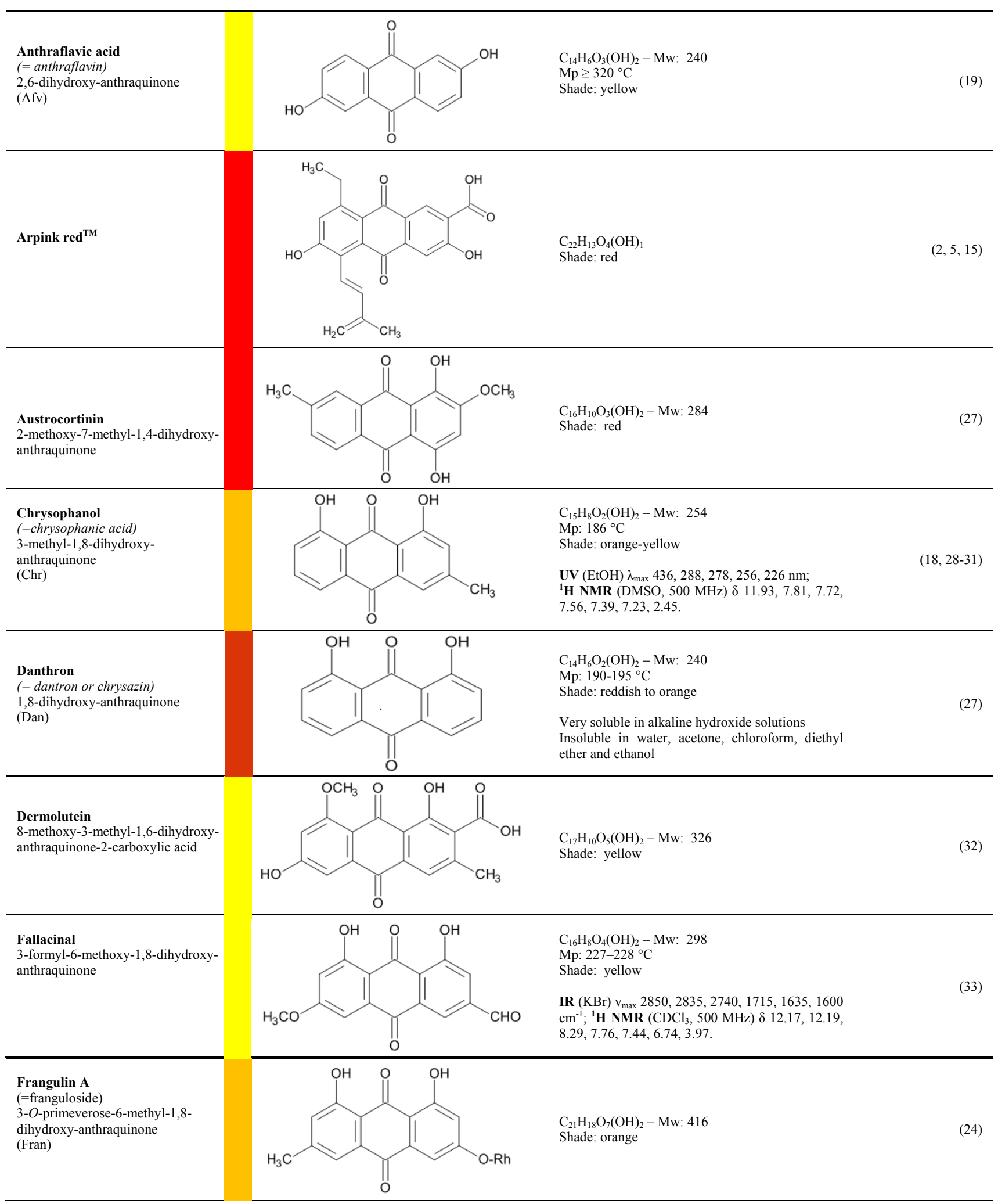

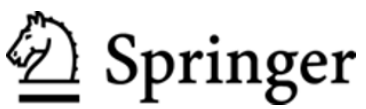




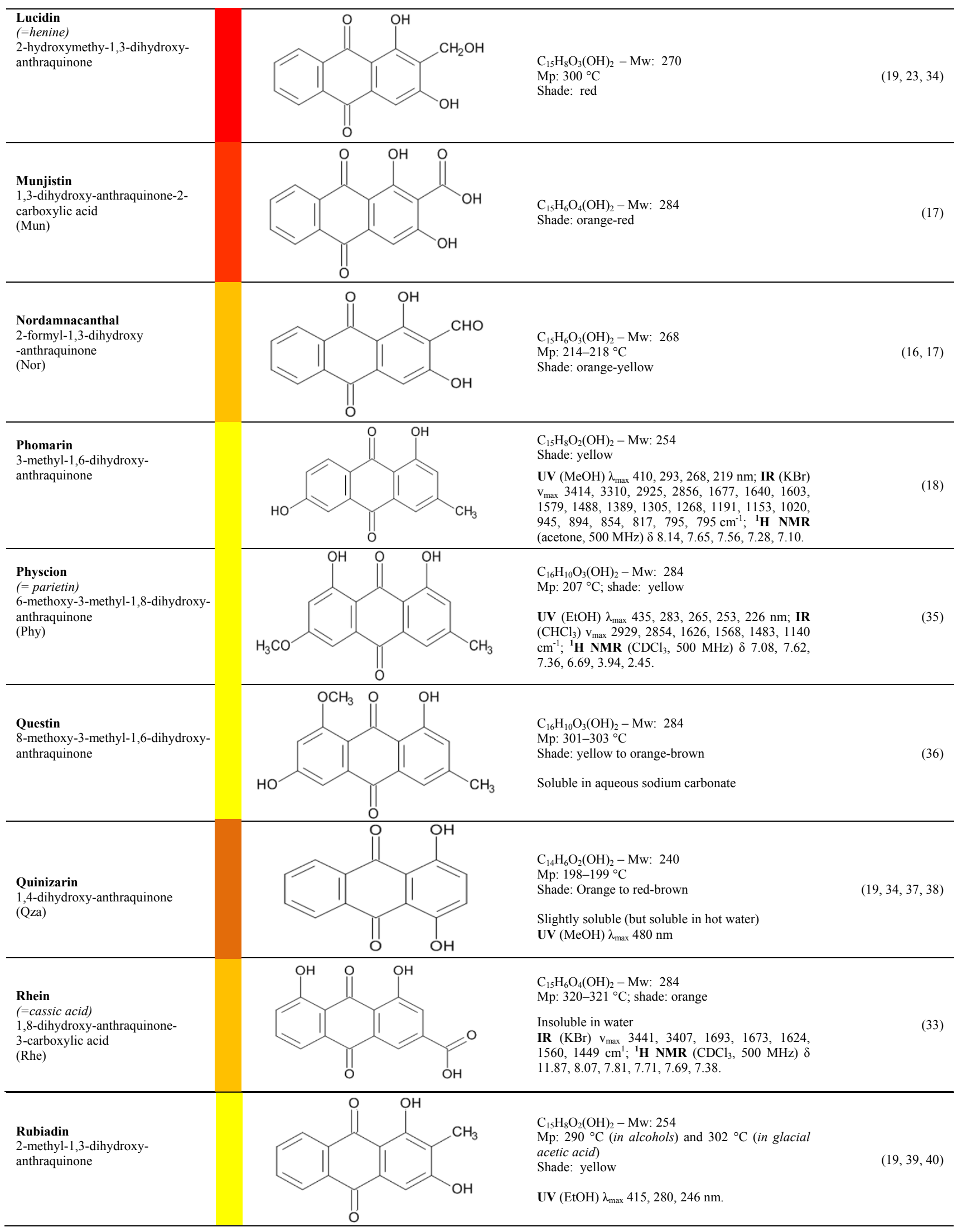




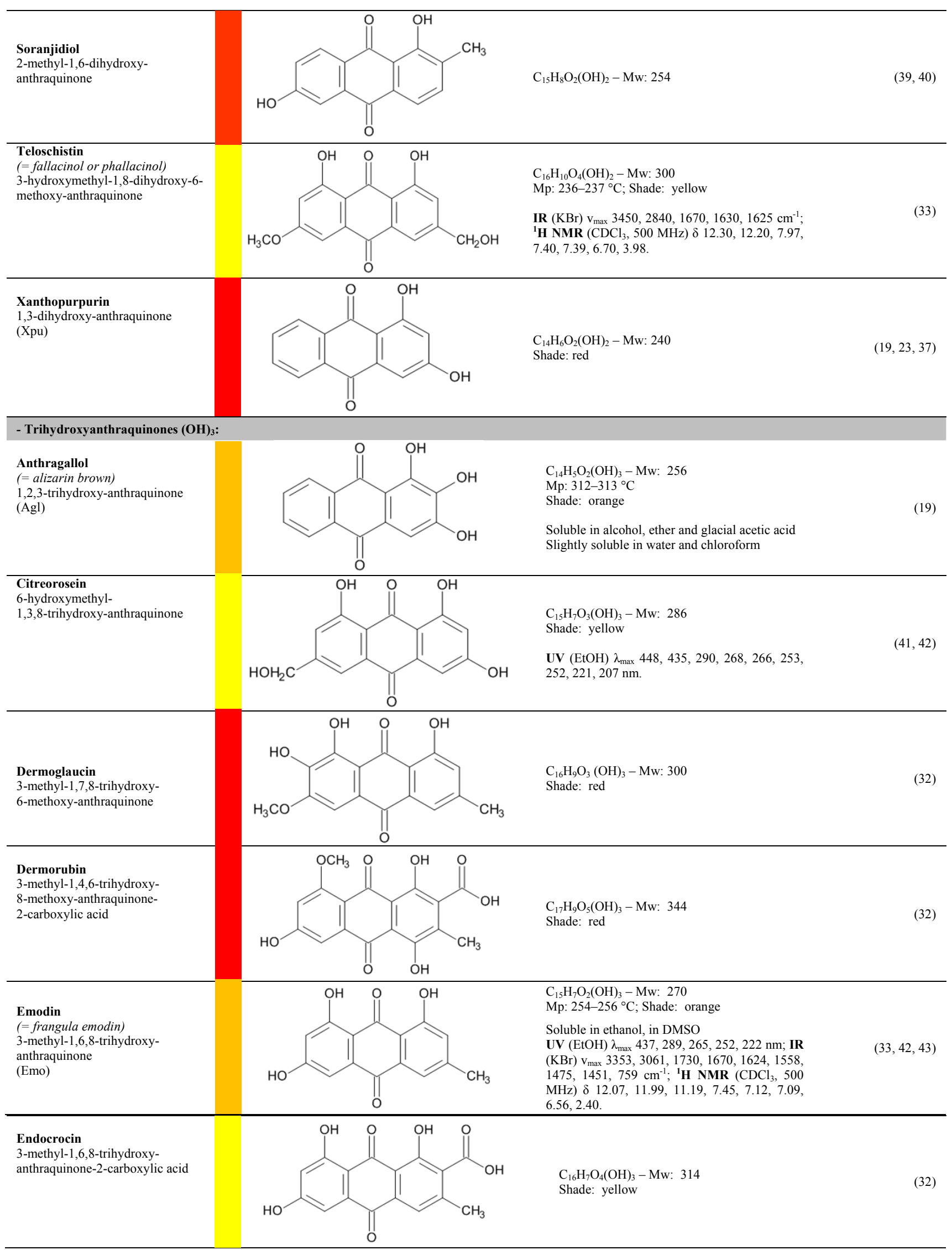

\section{型 Springer}




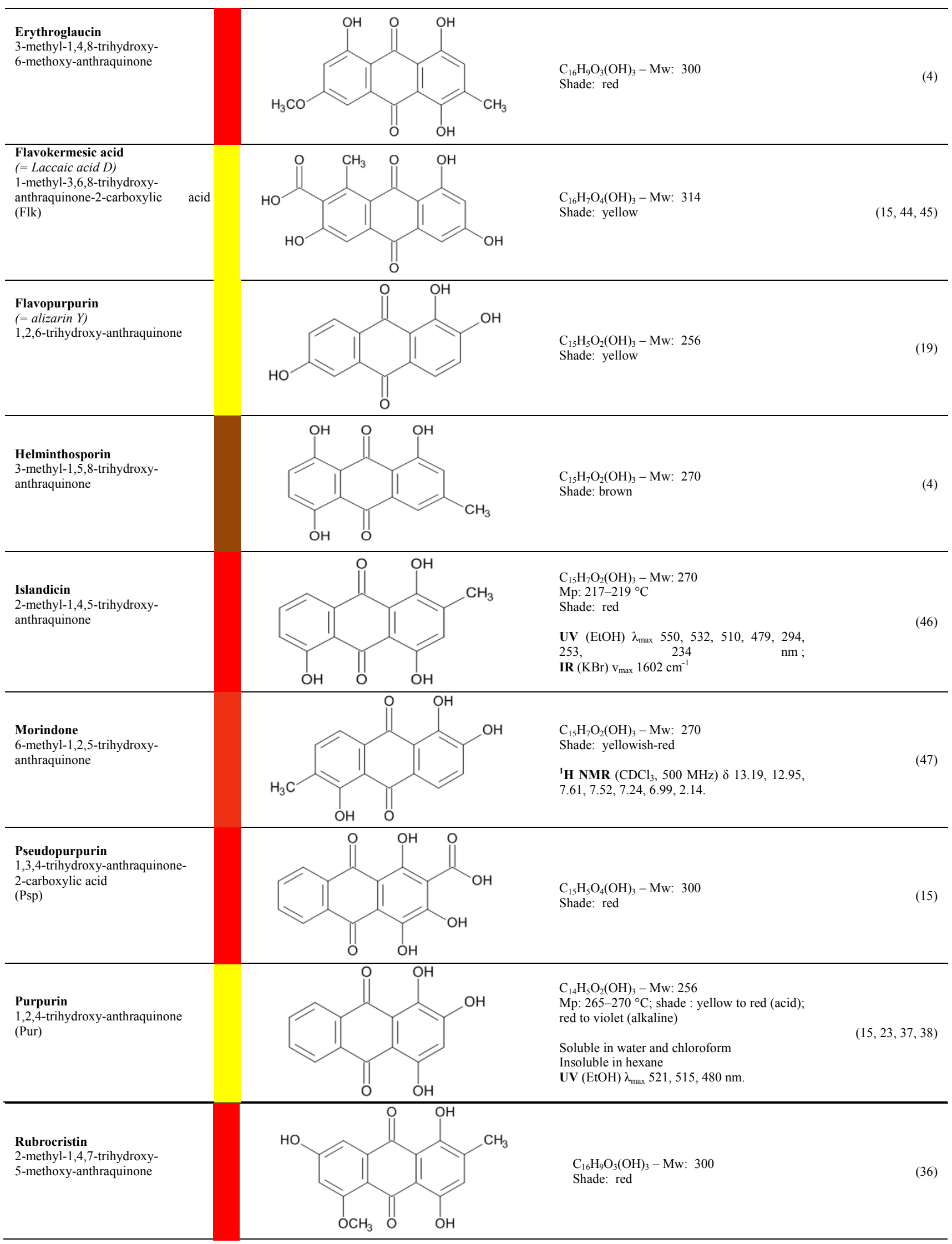




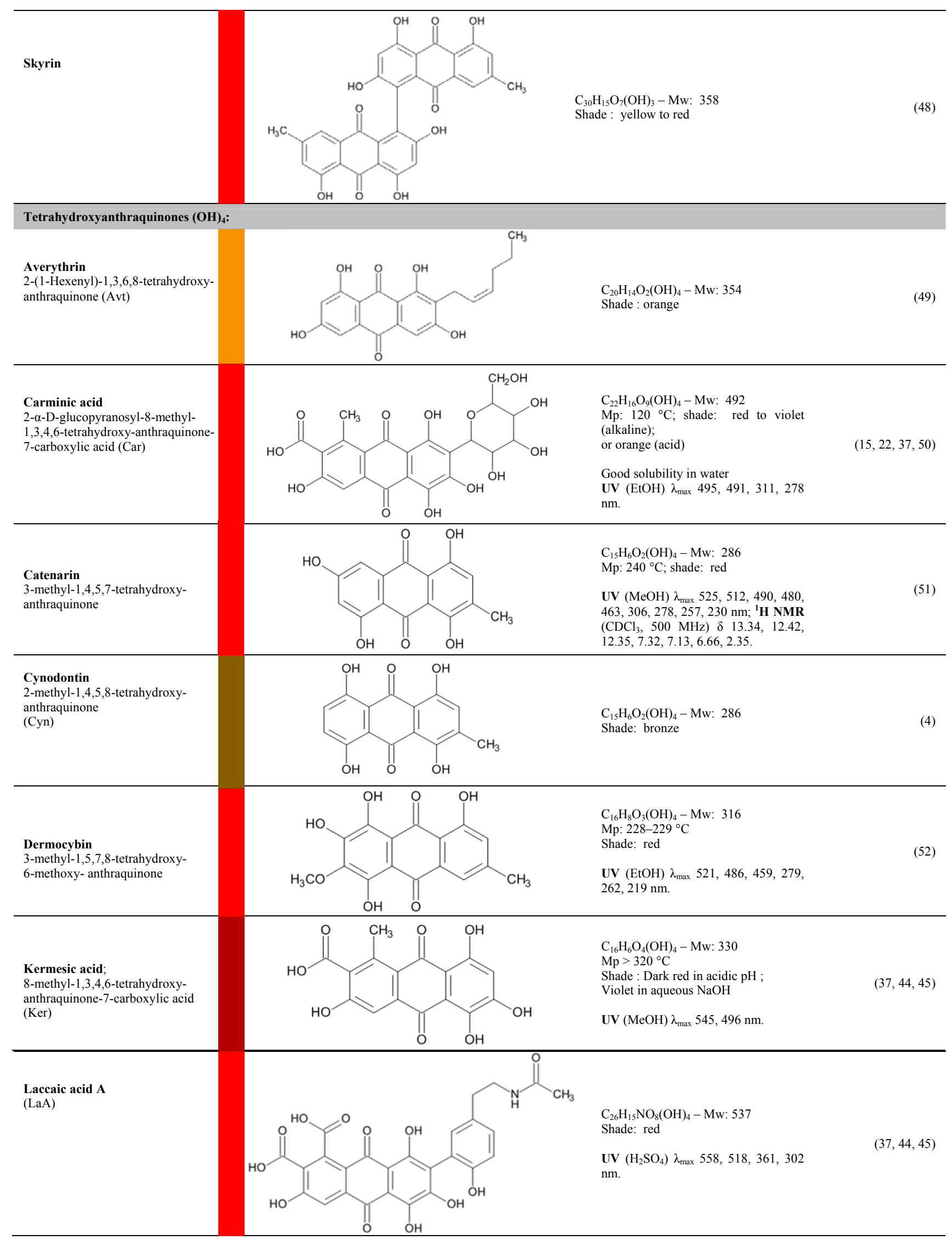

\section{黛 Springer}




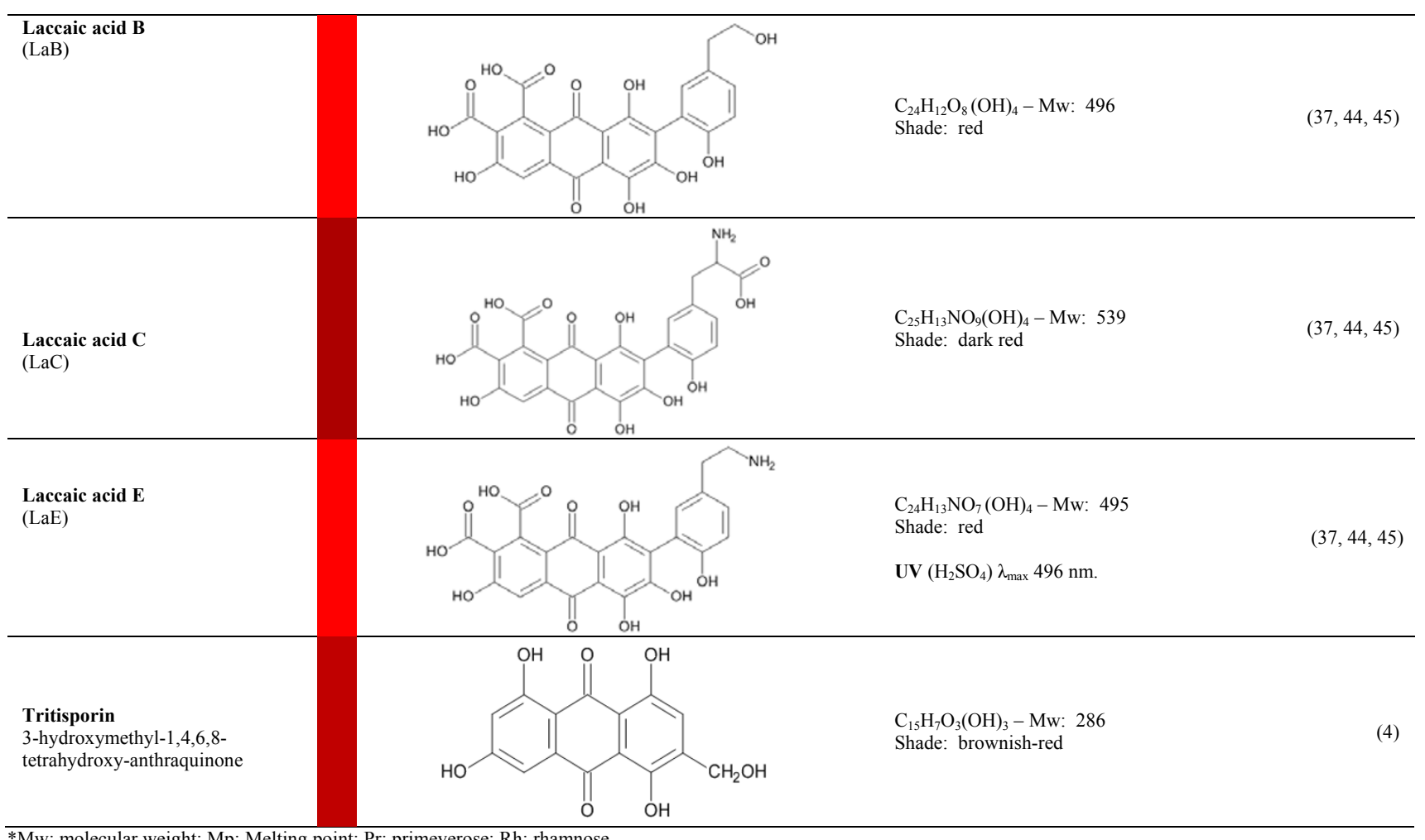

*Mw: molecular weight; Mp: Melting point; Pr: primeverose; Rh: rhamnose

(yellow(acid) to red(alkali)), pseudopurpurin (orange), purpurin (dark red) and lucidin (red). A practical HAQN classification, according to the respective biosynthetic pathway of the compound and the position of the functional groups added on the 9,10-anthraquinone skeleton, is shown on Table 3. This classification is partially based on that proposed by Rafaëlly et al. in $2008^{45}$. It appears that the natural HAQN pigments formed via the chorismate/o-succinylbenzoic acid pathway are all classified in the entire 'group E' of HAQN dyes because they have substitution only on one aromatic ring, like alizarin and purpurin. In contrast, the HAQN pigments that are synthesized via the polyketide pathway are classified in the 'group $\mathrm{A}_{1}$ ' (compounds show substitution on both aromatic rings and at least two hydroxyl groups in both $\mathrm{R} 1$ and R8 positions, like emodin, chrysophanol or physcion) or into the 'group $\mathrm{A}_{2}$ ' of HAQN dyes (compounds show substitution on both aromatic rings and at least two hydroxyl groups in R1 and R6 positions and one methoxyl group in R8 position, like dermolutein and dermorubin).

\section{The main natural sources of hydroxyanthraquinoid pigments}

The natural HAQN pigments are mainly found in plants like Rubiaceae, Polygonaceae, Rhamnaceae, Fabaceae, Liliaceae, Bignoniaceae and Pedaliaceae, in lichens and in the animal kingdom (insects).

\subsection{Hydroxyanthraquinoid pigments from plants}

In plants, the dyestuff is often extracted from dried roots. HAQN pigments are mostly present as sugar derivatives - the glycosides - but the free form - the aglycones - are widely distributed as well. For example, the European madder roots contain $2 \%-3.5 \%$ of the dry weight of di- and tri- hydroxyanthraquinone-glycosides and, in general for higher plants, the HAQN-based colorant content from the dry mass is often under $5 \%{ }^{15}$. The anthraquinone glycosides are formed when one or more sugar molecules, mostly glucose or rhamnose, are bound to the aglycone by a $\beta$-glycoside linkage to hydroxyl group at position C-8 (in the case of glucose) or the one at C-6 (in the case of rhamnose) $)^{58}$. During storage, hydrolysis of the glycosides occurs, which is completed under acidic conditions. In the literature, a total of more than 35 anthraquinoid compounds have been reported to be extracted from roots of European madder (Rubia tinctorum Linn., i.e. the most important species of the plant family Rubiaceae), even if a part of the compounds is believed to be artefacts formed during extraction or drying ${ }^{15}$. The main HAQN colouring compounds of plants of the Rubiaceae family (e.g. Rubia spp., Galium spp., Morinda spp., Hypericum spp., Polygonum spp. and Cinchona spp.) $)^{14,15,23,34,38-40,45,59-73}$ are alizarin (yellow to red, group $\mathrm{E}_{3}$ ), pseudopurpurin (orange, group $\mathrm{E}_{2}$ ), purpurin (dark red, group $\mathrm{E}_{2}$ ), lucidin-3-O-primeveroside (red, group $\mathrm{E}_{3}$ ), ruberythric acid (golden-yellow, group $\mathrm{E}_{3}$ ), nordamnacanthal (orange, group $\mathrm{E}_{3}$ ) and munjistin (orange-red, group $\mathrm{E}_{3}$ ) (see Table 4). These colouring compounds are all classified in the entire 'group E' of HAQN dyes and they are formed through the chorismate/o-succinylbenzoic acid pathway as mentioned above.

In contrast, in other higher plants such as the Polygonaceae

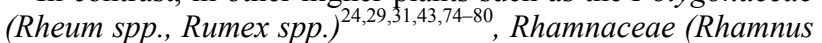
spp. $)^{25,81}$ Fabaceae (Cassia spp.) , $^{30,33,82}$, Liliaceae (Aloes spp. $)^{83,84}$ and Pedaliaceae (Ceratotheca spp. ${ }^{85}$ families, the most common naturally occurring HAQN pigments are synthesized via the polyketide pathway (Fig. 2). Relevant pigments are emodin (yellow), aloe-emodin (yellow), physcion (yellow), rhein (orange) and chrysophanol (orange-red) (see 
Table 4). These HAQN pigments are all classified in the 'group $\mathrm{A}_{1}$ ' of HAQN because they show substitution on both

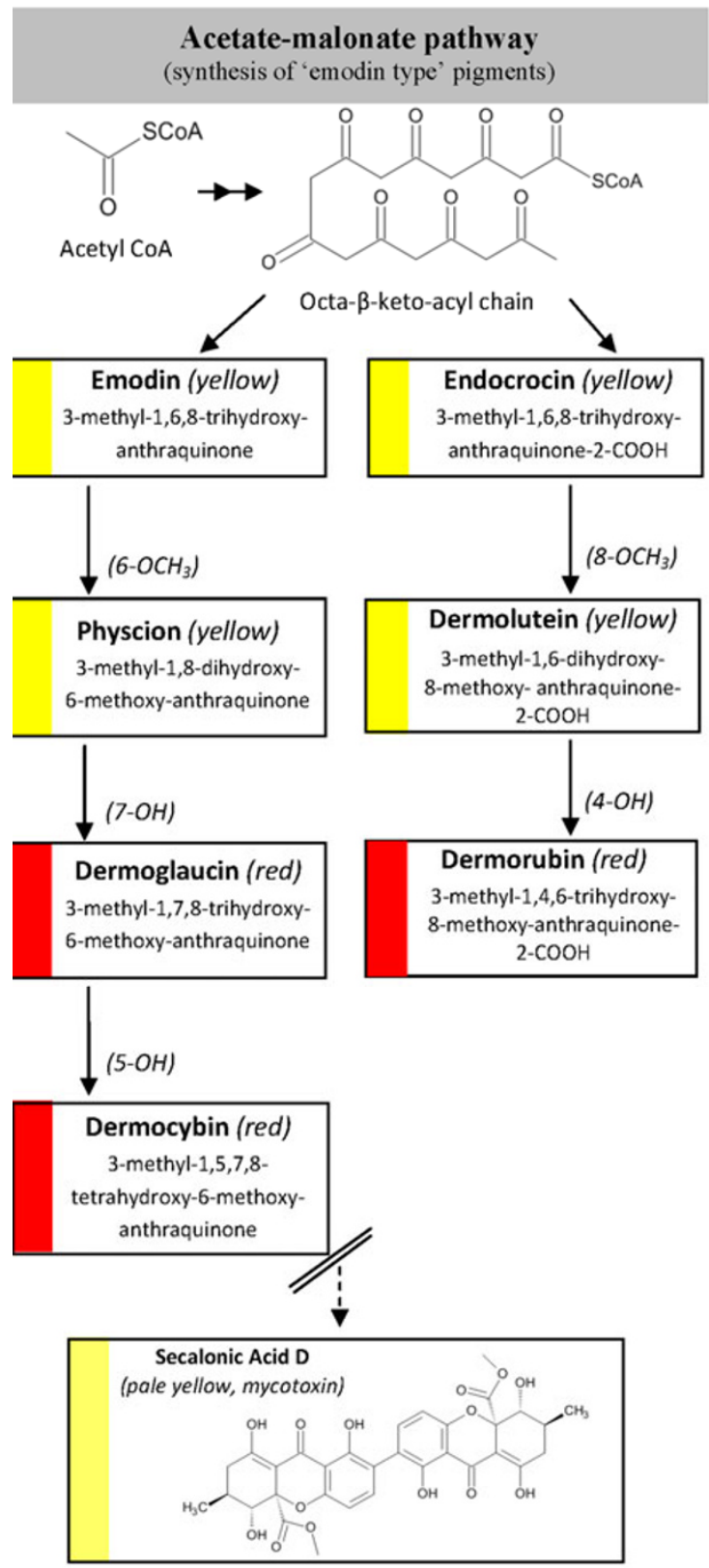

References: $(3,32,54)$

Characteristics of 'emodin type' pigments:

They show substitution on both aromatic rings and at least one hydroxyl group in position R1 and one hydroxyl (-OH) or methoxyl $\left(-\mathrm{OCH}_{3}\right)$ group in position $\mathrm{R} 8$

Main biological sources of 'emodin type' pigments:

Plants of the Fabaceae, Rhamnaceae and Polygonaceae (Cassia sp., Phamnus sp., Rheum sp., Rumex sp.):

Lichens: Caloplaca sp., Xanthoria sp., Teloschistes sp., Laurera sp., Nephroma sp., Heteroderma $s p$.

Fungi: Penicillium sp., Aspergillus sp., Trichoderma sp., Dermocybe $s p$. aromatic rings and at least two hydroxyl groups in both $\mathrm{R} 1$ and R8 positions.

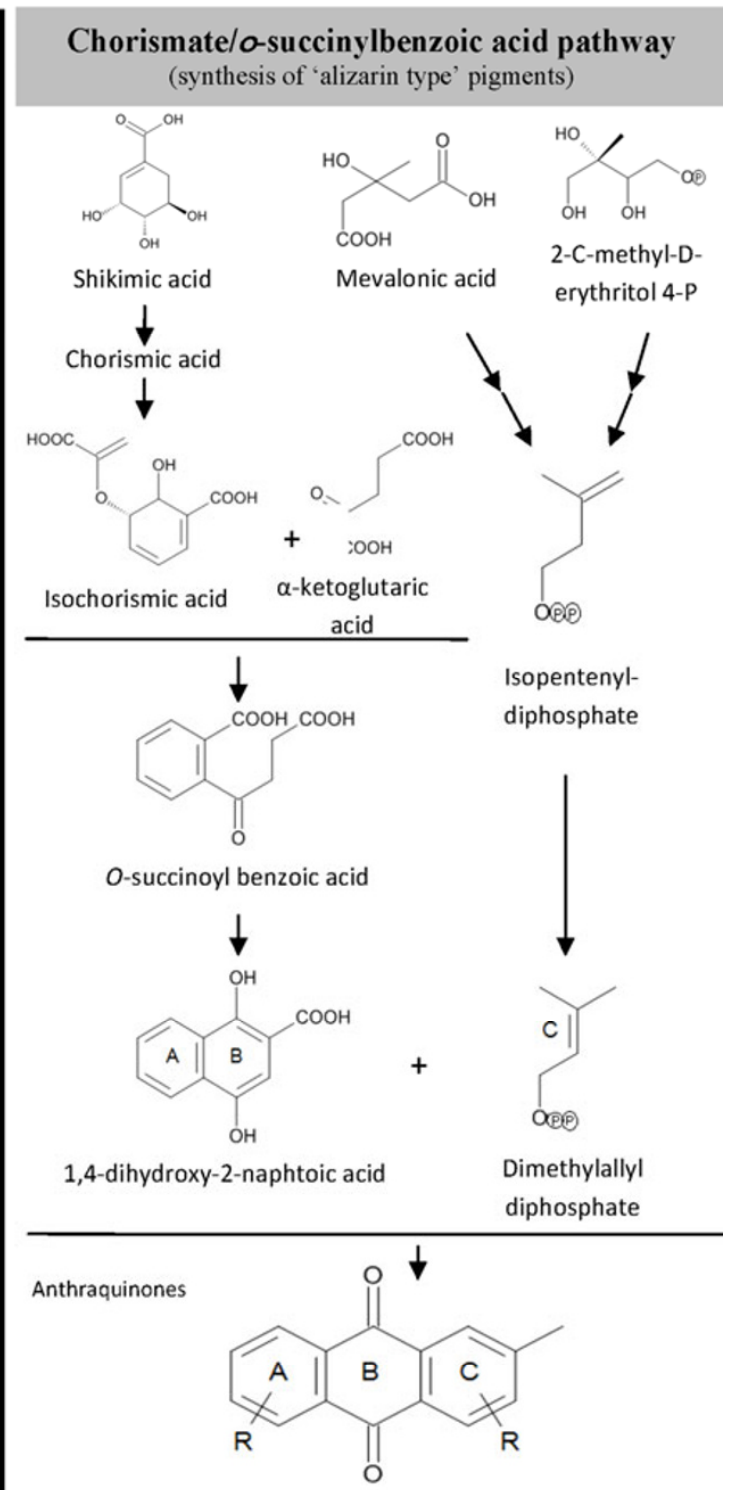

\begin{tabular}{|l|l|}
\hline $\begin{array}{l}\text { Alizarin (yellow to red) } \\
\text { 1,2-dihydroxy-anthraquinone }\end{array}$ & $\begin{array}{l}\text { Purpurin (dark red) } \\
\text { 1,2,4-trihydroxy-anthraquinone }\end{array}$ \\
\hline $\begin{array}{l}\text { Pseudopurpurin (orange) } \\
\text { 1,3,4-trihydroxy-anthraquinone- }\end{array}$ & $\begin{array}{l}\text { Lucidin (red) } \\
\text { 2-chydroxymethyl-1,3-dihydroxy- } \\
\text { anthraquinone }\end{array}$ \\
\hline
\end{tabular}

References: $(54,56,57)$

Characteristics of 'alizarin type' pigments:

They only have one of the rings unsubstituted and at least one hydroxyl group in position R1 (classified into the 'group E' of hydroxyanthraquinoid dyes)

Main biological sources of 'alizarin type' pigments:

Plants of the Rubiaceae family (Rubia sp., Galium $s p$. Morinda sp. and Cinchona sp.)

Figure 2. The two main biosynthetic pathways of hydroxyanthraquinoid (HAQN) pigments in organisms

\section{粤 Springer}


Table 3. Position of functional groups added on 9,10-anthraquinone skeleton in natural hydroxyanthraquinoid (HAQN) pigments and their classification into several groups

\begin{tabular}{|c|c|c|c|c|c|c|c|c|c|}
\hline HAQN compound & $\mathbf{R 1}$ & $\mathbf{R 2}$ & $\mathbf{R 3}$ & $\mathbf{R 4}$ & $\mathbf{R 5}$ & R6 & $\mathbf{R} 7$ & $\mathbf{R 8}$ & shade \\
\hline \multicolumn{10}{|c|}{ Group A1: at least two hydroxyl groups in both R1 and R8 positions: } \\
\hline Aloe-emodin & OH & $\mathrm{H}$ & $\mathrm{CH}_{2} \mathrm{OH}$ & $\mathrm{H}$ & $\mathrm{H}$ & $\mathrm{H}$ & $\mathrm{H}$ & ОH & orange \\
\hline Averythrin & $\mathbf{O H}$ & $\mathrm{C}_{6} \mathrm{H}_{11} \mathrm{H}$ & $\mathrm{OH}$ & $\mathrm{H}$ & $\mathrm{H}$ & $\mathrm{OH}$ & $\mathrm{H}$ & $\mathbf{O H}$ & orange \\
\hline Chrysophanol & OH & $\mathrm{H}$ & $\mathrm{CH}_{3}$ & $\mathrm{H}$ & $\mathrm{H}$ & $\mathrm{H}$ & $\mathrm{H}$ & ОH & orange-red \\
\hline Citreorosein & $\mathbf{O H}$ & $\mathrm{H}$ & $\mathrm{OH}$ & $\mathrm{H}$ & $\mathrm{H}$ & $\mathrm{CH}_{2} \mathrm{OH}$ & $\mathrm{H}$ & $\mathbf{O H}$ & yellow \\
\hline Cynodontin & $\mathbf{O H}$ & $\mathrm{H}$ & $\mathrm{CH}_{3}$ & $\mathrm{OH}$ & $\mathrm{OH}$ & $\mathrm{H}$ & $\mathrm{H}$ & $\mathbf{O H}$ & bronze \\
\hline Danthron (= chrysarin) & OH & $\mathrm{H}$ & $\mathrm{H}$ & $\mathrm{H}$ & $\mathrm{H}$ & $\mathrm{H}$ & $\mathrm{H}$ & OH & reddish to orange \\
\hline Dermoglaucin & $\mathbf{O H}$ & $\mathrm{H}$ & $\mathrm{CH}_{3}$ & $\mathrm{H}$ & $\mathrm{H}$ & $\mathrm{OCH}_{3}$ & $\mathrm{OH}$ & $\mathbf{O H}$ & red \\
\hline Dermocybin & OH & $\mathrm{H}$ & $\mathrm{CH}_{3}$ & $\mathrm{H}$ & $\mathrm{OH}$ & $\mathrm{OCH}_{3}$ & $\mathrm{OH}$ & OH & red \\
\hline Emodin & ОH & $\mathrm{H}$ & $\mathrm{CH}_{3}$ & $\mathrm{H}$ & $\mathrm{H}$ & $\mathrm{OH}$ & $\mathrm{H}$ & ОH & orange \\
\hline Endocrocin & OH & $\mathrm{COOH}$ & $\mathrm{CH}_{3}$ & $\mathrm{H}$ & $\mathrm{H}$ & $\mathrm{OH}$ & $\mathrm{H}$ & OH & yellow \\
\hline Erythroglaucin & OH & $\mathrm{H}$ & $\mathrm{CH}_{3}$ & $\mathrm{OH}$ & $\mathrm{H}$ & $\mathrm{OCH}_{3}$ & $\mathrm{H}$ & OH & red \\
\hline Fallacinal & OH & $\mathrm{H}$ & $\mathrm{CHO}$ & $\mathrm{H}$ & $\mathrm{H}$ & $\mathrm{OCH}_{3}$ & $\mathrm{H}$ & ОH & yellow \\
\hline Frangulin A & OH & $\mathrm{H}$ & $\mathrm{O}-\mathrm{Rh}$ & $\mathrm{H}$ & $\mathrm{H}$ & $\mathrm{CH}_{3}$ & $\mathrm{H}$ & OH & orange \\
\hline Helminthosporin & OH & $\mathrm{H}$ & $\mathrm{CH}_{3}$ & $\mathrm{H}$ & $\mathrm{OH}$ & $\mathrm{H}$ & $\mathrm{H}$ & OH & maroon \\
\hline Physcion (= parietin) & ОH & $\mathrm{H}$ & $\mathrm{CH}_{3}$ & $\mathrm{H}$ & $\mathrm{H}$ & $\mathrm{OCH}_{3}$ & $\mathrm{H}$ & ОH & yellow \\
\hline Rhein & $\mathbf{O H}$ & $\mathrm{H}$ & $\mathrm{COOH}$ & $\mathrm{H}$ & $\mathrm{H}$ & $\mathrm{H}$ & $\mathrm{H}$ & $\mathbf{O H}$ & orange \\
\hline Teloschistin $(=$ fallacinol $)$ & OH & $\mathrm{H}$ & $\mathrm{CH}_{2} \mathrm{OH}$ & $\mathrm{H}$ & $\mathrm{H}$ & $\mathrm{OCH}_{3}$ & $\mathrm{H}$ & ОH & yellow \\
\hline Tritisporin & $\mathbf{O H}$ & $\mathrm{H}$ & $\mathrm{CH}_{2} \mathrm{OH}$ & $\mathrm{OH}$ & $\mathrm{H}$ & $\mathrm{OH}$ & $\mathrm{H}$ & $\mathbf{O H}$ & reddish brown \\
\hline \multicolumn{10}{|c|}{ Group A2: at least two hydroxyl groups in R1 and R6 positions, and one methoxyl group in R8 position } \\
\hline Questin & OH & $\mathrm{H}$ & $\mathrm{CH}_{3}$ & $\mathrm{H}$ & $\mathrm{H}$ & OH & $\mathrm{H}$ & $\mathrm{OCH}_{3}$ & yellow to orange \\
\hline Dermolutein & ОH & $\mathrm{COOH}$ & $\mathrm{CH}_{3}$ & $\mathrm{H}$ & $\mathrm{H}$ & OH & $\mathrm{H}$ & $\mathrm{OCH}_{3}$ & yellow \\
\hline Dermorubin & $\mathbf{O H}$ & $\mathrm{COOH}$ & $\mathrm{CH}_{3}$ & $\mathrm{OH}$ & $\mathrm{H}$ & $\mathbf{O H}$ & $\mathrm{H}$ & $\mathrm{OCH}_{3}$ & red \\
\hline \multicolumn{10}{|c|}{ Group B: Four hydroxyl groups in $R 1, R 3, R 4$ and $R 6$ positions, and one carboxyl group in $R 7$ position: } \\
\hline Kermesic acid & $\mathbf{O H}$ & $\mathrm{H}$ & $\mathbf{O H}$ & $\mathrm{OH}$ & $\mathrm{H}$ & $\mathbf{O H}$ & $\mathrm{COOH}$ & $\mathrm{CH}_{3}$ & red \\
\hline Carminic acid & ОH & Glc & OH & OH & $\mathrm{H}$ & OH & СООН & $\mathrm{CH}_{3}$ & red \\
\hline Laccaic acid A & OH & $\mathrm{C}_{10} \mathrm{H}_{12} \mathrm{O}_{2} \mathrm{~N}$ & OH & OH & $\mathrm{H}$ & OH & $\mathrm{COOH}$ & $\mathrm{COOH}$ & red \\
\hline Laccaic acid B & OH & $\mathrm{C}_{8} \mathrm{H}_{9} \mathrm{O}_{2}$ & $\mathbf{O H}$ & ОH & $\mathrm{H}$ & ОH & $\mathrm{COOH}$ & $\mathrm{COOH}$ & red \\
\hline Laccaic acid C & OH & $\mathrm{C}_{9} \mathrm{H}_{10} \mathrm{O}_{3} \mathrm{~N}$ & ОH & ОH & $\mathrm{H}$ & ОН & $\mathrm{COOH}$ & $\mathrm{COOH}$ & red \\
\hline Laccaic acid E & OH & $\mathrm{C}_{8} \mathrm{H}_{10} \mathrm{ON}$ & OH & OH & $\mathrm{H}$ & ОH & $\mathrm{COOH}$ & $\mathrm{COOH}$ & red \\
\hline \multicolumn{10}{|c|}{ Group C1: at least two hydroxyl groups in $\mathrm{R} 1$ and $\mathrm{R} 4$ positions, and at least one functional group (-OH, $\left.-\mathrm{CH}_{3}\right)$ in $\mathrm{R}^{7}$ position: } \\
\hline Austrocortinin & OH & $\mathrm{OCH}_{3}$ & $\mathrm{H}$ & $\mathbf{O H}$ & $\mathrm{H}$ & $\mathrm{H}$ & $\mathrm{CH}_{3}$ & $\mathrm{H}$ & red \\
\hline Catenarin & OH & $\mathrm{H}$ & $\mathrm{CH}_{3}$ & ОH & $\mathrm{OH}$ & $\mathrm{H}$ & $\mathrm{OH}$ & $\mathrm{H}$ & red \\
\hline Rubrocristin & OH & $\mathrm{CH}_{3}$ & $\mathrm{H}$ & OH & $\mathrm{OCH}_{3}$ & $\mathrm{H}$ & $\mathrm{OH}$ & $\mathrm{H}$ & red \\
\hline \multicolumn{10}{|c|}{ Group C2: at least two hydroxyl groups in $R 1$ and $R 6$ positions, and position $R 8$ unsubstituted: } \\
\hline Flavopurpurin & OH & $\mathrm{OH}$ & $\mathrm{H}$ & $\mathrm{H}$ & $\mathrm{H}$ & OH & $\mathrm{H}$ & $\mathrm{H}$ & yellow \\
\hline Phomarin & OH & $\mathrm{H}$ & $\mathrm{CH}_{3}$ & $\mathrm{H}$ & $\mathrm{H}$ & $\mathbf{O H}$ & $\mathrm{H}$ & $\mathrm{H}$ & yellow \\
\hline Soranjidiol & $\mathbf{O H}$ & $\mathrm{CH}_{3}$ & $\mathrm{H}$ & $\mathrm{H}$ & $\mathrm{H}$ & $\mathbf{O H}$ & $\mathrm{H}$ & $\mathrm{H}$ & yellowish-red \\
\hline \multicolumn{10}{|c|}{ Group C3: at least two hydroxyl groups in $R 1$ and $R 5$ positions, and position $R 8$ unsubstituted: } \\
\hline Islandicin & OH & $\mathrm{CH}_{3}$ & $\mathrm{H}$ & $\mathrm{OH}$ & OH & $\mathrm{H}$ & $\mathrm{H}$ & $\mathrm{H}$ & red \\
\hline Morindone & $\mathbf{O H}$ & $\mathrm{OH}$ & $\mathrm{H}$ & $\mathrm{H}$ & $\mathbf{O H}$ & $\mathrm{CH}_{3}$ & $\mathrm{H}$ & $\mathrm{H}$ & yellowish-red \\
\hline \multicolumn{10}{|c|}{ Group D: no hydroxyl group in R1 position, at least one hydroxyl group in R2 position, and position R8 unsubstituted: } \\
\hline Anthraflavic acid (= anthraflavin) & $\mathrm{H}$ & OH & $\mathrm{H}$ & $\mathrm{H}$ & $\mathrm{H}$ & $\mathrm{OH}$ & $\mathrm{H}$ & $\mathrm{H}$ & yellow \\
\hline Skyrin & $\mathrm{C}_{15} \mathrm{H}_{9} \mathrm{O}_{5}$ & OH & $\mathrm{H}$ & $\mathrm{OH}$ & $\mathrm{OH}$ & $\mathrm{H}$ & $\mathrm{CH}_{3}$ & $\mathrm{H}$ & yellow to red \\
\hline
\end{tabular}

Compounds with functional groups only on one aromatic ring (group E):

Group E1: no hydrogen intramolecular bonds with carbonyl function:

\begin{tabular}{|c|c|c|c|c|c|c|c|c|c|}
\hline 3-MeO-hystazarin & $\mathrm{H}$ & $\mathrm{OH}$ & $\mathrm{OCH}_{3}$ & $\mathrm{H}$ & $\mathrm{H}$ & $\mathrm{H}$ & $\mathrm{H}$ & $\mathrm{H}$ & \\
\hline Damnacanthal & $\mathrm{OCH}_{3}$ & $\mathrm{CHO}$ & $\mathrm{OH}$ & $\mathrm{H}$ & $\mathrm{H}$ & $\mathrm{H}$ & $\mathrm{H}$ & $\mathrm{H}$ & pale yellow \\
\hline \multicolumn{10}{|c|}{ Group E2: at least 2 hydroxyl groups in $R 1$ and $R 4$ positions: } \\
\hline Purpurin & OH & $\mathrm{OH}$ & $\mathrm{H}$ & OH & $\mathrm{H}$ & $\mathrm{H}$ & $\mathrm{H}$ & $\mathrm{H}$ & dark red \\
\hline Pseudopurpurin & OH & $\mathrm{COOH}$ & $\mathrm{OH}$ & OH & $\mathrm{H}$ & $\mathrm{H}$ & $\mathrm{H}$ & $\mathrm{H}$ & orange \\
\hline Quinizarin & $\mathbf{O H}$ & $\mathrm{H}$ & $\mathrm{H}$ & $\mathbf{O H}$ & $\mathrm{H}$ & $\mathrm{H}$ & $\mathrm{H}$ & $\mathrm{H}$ & orange-red \\
\hline \multicolumn{10}{|c|}{ Group E3: 2 or 3 hydroxyl groups but always one in position $R 1$ and none in position $R 4$ : } \\
\hline Alizarin & OH & $\mathrm{OH}$ & $\mathrm{H}$ & $\mathbf{H}$ & $\mathrm{H}$ & $\mathrm{H}$ & $\mathrm{H}$ & $\mathrm{H}$ & yellow to red \\
\hline Anthragallol & OH & $\mathrm{OH}$ & $\mathrm{OH}$ & $\mathbf{H}$ & $\mathrm{H}$ & $\mathrm{H}$ & $\mathrm{H}$ & $\mathrm{H}$ & orange \\
\hline Lucidin-3-O-primeveroside & OH & $\mathrm{CH}_{2} \mathrm{OH}$ & $\mathrm{O}-\mathrm{Pr}$ & $\mathbf{H}$ & $\mathrm{H}$ & $\mathrm{H}$ & $\mathrm{H}$ & $\mathrm{H}$ & red \\
\hline Lucidin (= henine) & OH & $\mathrm{CH}_{2} \mathrm{OH}$ & $\mathrm{OH}$ & $\mathbf{H}$ & $\mathrm{H}$ & $\mathrm{H}$ & $\mathrm{H}$ & $\mathrm{H}$ & red \\
\hline Munjistin & OH & $\mathrm{COOH}$ & $\mathrm{OH}$ & $\mathbf{H}$ & $\mathrm{H}$ & $\mathrm{H}$ & $\mathrm{H}$ & $\mathrm{H}$ & orange-red \\
\hline Nordamnacanthal & OH & $\mathrm{CHO}$ & $\mathrm{OH}$ & $\mathbf{H}$ & $\mathrm{H}$ & $\mathrm{H}$ & $\mathrm{H}$ & $\mathrm{H}$ & orange-yellow \\
\hline Pachybasin & OH & $\mathrm{CH}_{3}$ & $\mathrm{H}$ & $\mathbf{H}$ & $\mathrm{H}$ & $\mathrm{H}$ & $\mathrm{H}$ & $\mathrm{H}$ & yellow \\
\hline Ruberythric acid & OH & $\mathrm{O}-\mathrm{Pr}$ & $\mathrm{H}$ & $\mathbf{H}$ & $\mathrm{H}$ & $\mathrm{H}$ & $\mathrm{H}$ & $\mathrm{H}$ & golden-yellow \\
\hline Rubiadin & OH & $\mathrm{CH}_{3}$ & $\mathrm{OH}$ & $\mathbf{H}$ & $\mathrm{H}$ & $\mathrm{H}$ & $\mathrm{H}$ & $\mathrm{H}$ & yellow \\
\hline Xanthopurpurin & OH & $\mathrm{H}$ & $\mathrm{OH}$ & $\mathbf{H}$ & $\mathrm{H}$ & $\mathrm{H}$ & $\mathrm{H}$ & $\mathrm{H}$ & red \\
\hline
\end{tabular}

Pr: primeverose; Glc: glucose; Rh: rhamnose 
Table 4. Natural occurrence of hydroxyanthraquinoid (HAQN) pigments in plants

\begin{tabular}{|c|c|c|}
\hline Latin name & Main colouring components & References \\
\hline \multicolumn{3}{|l|}{ Rubiaceae } \\
\hline $\begin{array}{l}\text { Rubia tinctorum } L . \\
\text { (= European madder) }\end{array}$ & $\begin{array}{l}\text { alizarin; purpurin; pseudopurpurin; lucidin; rubiadin; xanthopurpurin; munjinstin; anthraflavin; quinizarin; } \\
\text { danthron; anthragallol; nordamnacanthal; ruberythric acid; lucidin primeveroside, alizarin-2-methyl ether; } \\
\text { lucidin- } \omega \text {-ethyl-ether; munjistin ethyl ether }\end{array}$ & $(23,34,38,59)$ \\
\hline Rubia cordifolia $L$. & rubiadin; alizarin; purpurin; pseudopurpurin; lucidin; munjinstin; xanthopurpurin; tectoquinone & $(14,15,23,60)$ \\
\hline Rubia akane & purpurin; ruberythric acid & $(15,45)$ \\
\hline Rubia peregrina $\mathrm{L}$. & pseudopurpurin & $(15)$ \\
\hline Galium aparine $\mathrm{L}$. & nordamnacanthal; xanthopurpurin; rubiadin & $(61)$ \\
\hline Galium sinaicum & $\begin{array}{l}\text { 7-methyl-anthragallol-1,3-dimethyl ether; 7-methyl-anthragallol-2-methyl ether; 6-methyl-anthragallol-3-methyl ether; } \\
\text { 8-hydroxy-anthragallol-2,3-dimethyl ether; 7-formyl-anthragallol-1,3-dimethylether; 6-hydroxy-xanthopurpurin; } \\
\text { 6-methoxy-lucidin- } \omega \text {-ethyl ether; copareolatin; copareolatin-6,7-dimethyl ether; copareolatin-5,7-dimethyl ether }\end{array}$ & $(62)$ \\
\hline $\begin{array}{l}\text { Galium verum } \mathrm{L} \text {. } \\
\text { (= Lady's bedstraw) }\end{array}$ & $\begin{array}{l}\text { alizarin; 1,3-dihydroxy-2-methoxymethyl; 1,3-dimethoxy-2-hydroxy,1,3-dihydroxy-2-acetoxy; 1-hydroxy-2-hydroxymethyl; } \\
\text { 1,3-dihydroxy-2-methyl; 1-methoxy-2-hydroxyanthraquinones; 1,3-dihydroxy-2-hydroxymethyl-6-methoxy anthraquinones }\end{array}$ & $(15,63)$ \\
\hline Galium mollugo L. & pseudopurpurin & $(15)$ \\
\hline \multirow[t]{2}{*}{ Galium spurium } & 8-hydroxy-3-methoxy-7-methyl-1,2-methylenedioxy-anthraquinone; & $(64)$ \\
\hline & 2,8-dihydroxy-1,3-dimethoxy-7-methyl-anthraquinone & \\
\hline Morinda officinalis & $\begin{array}{l}\text { alizarin; purpurin; pseudopurpurin; lucidin; rubiadin; 2-hydroxy-1-methoxy-anthraquinone; } \\
\text { 1,3,8-trihydroxy-2-methyl-anthraquinone }\end{array}$ & $(40)$ \\
\hline Morinda elliptica & $\begin{array}{l}\text { alizarin; purpurin; pseudopurpurin; lucidin; rubiadin; moridone; soranjidol; nordamnacanthal; } \\
\text { alizarin-1-methylether; lucidin- } \omega \text {-methylether }\end{array}$ & (39) \\
\hline Morinda citrifolia & damnacanthal; morindone; morindin; alizarin; physcion; morenone; morenone; ruberythric acid; rubiadin; lucidin & (65) \\
\hline Cinchona ledgeriana & $\begin{array}{l}\text { purpurin; rubiadin; anthragallol-1,2-dimethylether; anthragallol-1,3-dimethylether; 1-hydroxy-2-hydroxymethylanthraquinone; } \\
\text { 1-hydroxy-2-methylanthraquinone; morindone-5-methylether (or 1,7-dihydroxy-8-methoxy-2-methylanthraquinone) }\end{array}$ & $(66,67)$ \\
\hline Cinchona pubescens & $\begin{array}{l}\text { purpurin; alizarin-2-methylether; anthragallol-1,2-dimethylether;purpurin-1-methylether; } \\
\text { 1-hydroxy-2-hydroxymethyl-anthraquinone; 2-hydroxy-1,3,4-trimethoxy-anthraquinone }\end{array}$ & $(68)$ \\
\hline Cinchona succirubra & emodin; anthrapurpurin; quinizarin; 2,6-dihydroxyanthraquinone; 1,8-dihydroxyanthraquinone & (69) \\
\hline Cinchona robusta & robustaquinones $(\mathrm{A}-\mathrm{H}) ; 1,3,8$-trihydroxy-2-methyl anthraquinone; copareolatin 6-methylether & $(70)$ \\
\hline Asperula tinctoria $\mathrm{L}$. & alizarin; rubiadin & $(15,45)$ \\
\hline Asperula arvensis $\mathrm{L}$. & alizarin & (45) \\
\hline Oldenlandia umbellata $\mathrm{L}$. & $\begin{array}{l}\text { alizarin;1,2,3-trimethoxyanthraquinone; 3-MeO-hystazarin, ruberythric acid; 1,3-dimethoxy-2-hydroxyanthraquinone; } \\
\text { 1,2-dimethoxyanthraquinone; 1-methoxy-2-hydroxyanthraquinone;1,2-dihydroxyanthraquinone }\end{array}$ & $(45,71,72)$ \\
\hline Hedyotis auricularia $\mathrm{L}$ & alizarin & $(45)$ \\
\hline Crucianella maritima $\mathrm{L}$. & alizarin; 3-formyl-1-hydroxy-2-methoxy anthraquinone; alizarin-1-methyl ether; 1,4-dihydroxy-2-methoxy-anthraquinone & $(45,73)$ \\
\hline Coprosma lucida & anthragallol; lucidin; rubiadin & $(45)$ \\
\hline Hymenodictyon excelsum & anthragallol & $(45)$ \\
\hline \multicolumn{3}{|l|}{ Polygonaceae } \\
\hline Rheum officinale & emodin; chrysophanol; rhein & $(43,74)$ \\
\hline Rheum palmatum & chrysophanol; aloe-emodin; rhein; physcion; citreorosein & $(24,75)$ \\
\hline Rheum emodi & emodin; chrysophanol; aloe-emodin; rhein; physcion & $(29,76)$ \\
\hline Rheum rhabarbarum & emodin; chrysophanol; aloe-emodin; rhein; physcion & (31) \\
\hline Rumex dentatus & chrysophanol; physcion & (24) \\
\hline Rumex crispus & chrysophanol; parietin & $(77,78)$ \\
\hline Rumex acetosa & chrysophanol; physcion; emodin; emodin- $8-O-\beta$-D-glucopyranoside & (79) \\
\hline Rumex obtusifolius & aloe-emodin; chrysophanol; emodin & $(80)$ \\
\hline Rumex spp. (19 spp.) & emodin; chrysophanol; physcion; aloe-emodin; rhein & $(80)$ \\
\hline \multicolumn{3}{|l|}{ Rhamnaceae } \\
\hline Rhamnus saxatilis & emodin; chrysophanol; aloe-emodin; rhein; physcion & $(25)$ \\
\hline Rhamnus alpinus $\mathrm{L}$. & aloe-emodin; rhein; emodin; chrysophanol; physcion & $(81)$ \\
\hline \multicolumn{3}{|l|}{ Fabaceae } \\
\hline Cassia occidentalis $\mathrm{L}$. & emodin, chrysophanol, aloe-emodin, rhein, physcion & (30) \\
\hline Cassia tora & emodin; rhein; physcion & (33) \\
\hline Senna alata & aloe-emodin; emodin; rhein; chrysophanol & $(82)$ \\
\hline \multicolumn{3}{|l|}{ Liliaceae } \\
\hline Aloes spp. (32 spp.) & chrysophanol; asphodelin; chrysophanol-8-methyl ether; aloechrysone; helminthosporin; aloesaponols; aloesaponarins & $(83,84)$ \\
\hline \multicolumn{3}{|l|}{ Bignoniaceae } \\
\hline Tecoma ipes & tectoquinone & (45) \\
\hline \multicolumn{3}{|l|}{ Pedaliaceae } \\
\hline Ceratotheca triloba (Bernh.) & 1-hydroxy-4-methylanthraquinone & $(85)$ \\
\hline
\end{tabular}

\section{算 Springer}




\subsection{Hydroxyanthraquinoid pigments from lichens}

HAQN pigments found in some lichens are synthesized via the polyketide pathway. For example, the main colouring compounds in the lichens of the family Teloschistaceae

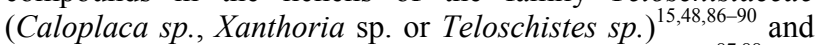
the family Trypetheliaceae (Laurera benguelensis) ${ }^{87,88}$ are emodin, physcion, teloschistin (yellow, group $\mathrm{A}_{1}$ ) and fallacinal (yellow, group $A_{1}$ ) (see Table 5). The lichens Nephroma laevigatum and Heteroderma obscurata also contain emodin ${ }^{91}$ whereas skyrin (i.e. a yellow to red pigment classified in the 'group D' of HAQN which show substitution on both aromatic rings; see Table 3 ) is the main component of Cladonia species $^{48}$.

\subsection{Hydroxyanthraquinoid pigments from insects}

In animals, HAQN-type pigments are known to be present only in a few insect species (see Table 5). Concerning the red carminic acid, kermesic acid and laccaic acid obtained from cochineal (Dactylopius coccus) $)^{15,22,37,50}$, kermes (Kermes vermilio $^{15,44}$ and lac (Kerria lacca $)^{15,44,45}$, respectively, they contain functional groups on both aromatic rings and particularly four hydroxyl groups in R1, R3, R4 and R6 positions, and one carboxyl group in $\mathrm{R} 7$ position. So these animal anthraquinoid glycosides are all classified in the 'group B' of HAQN (see Table 3). In both cochineal and kermes the pigments were obtained from the body and eggs of the female insect. Although the various species of the genus Porphyrophora, e.g. Armenian cochineal (P. hamelli) and Polish cochineal ( $P$. polonica), also contain carminic acid, dried specimens of Dactylopius coccus have a much higher content $(15 \%-20 \%)$ of carminic acid, compared with only $0.8 \%$ and $0.6 \%$ for the Armenian and Polish ones, respectively ${ }^{15,44,45}$. Lac insects of the Kerria family (e.g.
Kerria lacca and $K$. chinensis) contain mainly laccaic acids like laccaic acid A.

\subsection{Hydroxyanthraquinoid pigments from fungi}

HAQN pigments are widespread in nature and have been also found abundantly in microorganisms, particularly in filamentous fungi belonging to Penicillium spp. and Aspergillus spp., with different shades (see Table 6). For example, the pigment emodin was isolated from strains of Penicillium citrinum and P. islandicum ${ }^{5,92}$. The natural food colorant Arpink red ${ }^{\mathrm{TM}}$ manufactured by the Ascolor Biotech Czech company was claimed to be produced by fermentation and bioprocess engineering using the strain Penicillium oxalicum var. Armeniaca CCM 8242 obtained from soil ${ }^{5,92}$. On the second day of cultivation of this fungus in liquid broth containing carbohydrates, zinc sulfate and magnesium sulfate, a red colorant is released in the medium, increasing up to 1.5$2.0 \mathrm{~g} / \mathrm{L}$ of broth after 3-4 days ${ }^{2}$. After biosynthesis of the red colorant, the liquid is separated from the biomass by centrifugation or filtration. The liquid is then acidified to $\mathrm{pH}$ $3.0-2.5$ to precipitate the colorant. The precipitate is dissolved in ethyl alcohol and filtered. Following removal of alcohol, the colorant is obtained in the crystalline form as a dark red powder. In strains of Penicillium purpurogenum ${ }^{93}$, a red pigment of HAQN-type (none completely characterized) was also observed.

Some strains of Aspergillus (A. glaucus, A. cristatus and A. repens) $)^{4,5,35,36,92-94}$ were found to produce known yellow and red HAQN compounds such as emodin (yellow, group $\mathrm{A}_{1}$ ), physcion (yellow, group $A_{1}$ ), questin (yellow to orange-brown, group $A_{2}$ ), erythroglaucin (red, group $A_{1}$ ), catenarin (red, group $\mathrm{C}_{1}$; see Table 3 ) and rubrocristin (red, group $\mathrm{C}_{1}$; see Table 3). However, by using Penicillium or Aspergillus strains,

Table 5. Natural occurrence of hydroxyanthraquinoid (HAQN) pigments in lichens and in insects

\begin{tabular}{|c|c|c|c|}
\hline Dye source & Latin name & Main colouring components & References \\
\hline \multicolumn{4}{|l|}{ Lichens: } \\
\hline \multirow[t]{9}{*}{ Teloschistaceae } & Xanthoria spp. & physcion; emodin; parietin; fallacinal; teloschistin; citreorosein; erythroglaucin; fallacinol & $(15,48,86,87)$ \\
\hline & Xanthoria parietina $L$. & physcion; fallacinol; fallacinal; emodin; parietic acid & $(88)$ \\
\hline & Xanthoria mandschurica & erythroglaucin; parietin & $(89)$ \\
\hline & Xanthoria fallax & fallacinal; emodin; fallacinol; erythroglaucin; parietin & $(89)$ \\
\hline & Caloplaca spp. & 2-chloroemodin; citreorosein; emodin; fallacinal; parietin; physcion; teloschistin & $(48,87)$ \\
\hline & Caloplaca cerina & emodin; fallacinal; physcion; teloschistin & (33) \\
\hline & Caloplaca erythrantha & emodin; 7-chloroemodin & $(86)$ \\
\hline & Teloschistes exilis & parietin; teloschistin & $(86)$ \\
\hline & Teloschistes spp.(29 spp.) & parietin; emodin; teloschistin; fallacinal; parietic acid;erythroglaucin & $(90)$ \\
\hline Trypetheliaceae & Laurera benguelensis & parietin; physcion; citreorosein; emodin; fallacinal; teloschistin & $(87,88)$ \\
\hline Nephromataceae & Nephroma laevigatum & $\begin{array}{l}\text { emodin; 7-chloroemodin; 7-chloro-1- } O \text {-methylemodin;5-chloro- } \omega \text {-hydroxyemodin } \\
\text { 7-chloro-1- } O \text {-methyl- } \omega \text {-hydroxyemodin; 5-chloroemodin;5-chloro-1-O-methylemodin; } \\
\text { 5-chloro-1- } O \text {-methyl- } \omega \text {-hydroxyemodin; }\end{array}$ & $(91)$ \\
\hline Physciaceae & Heteroderma obscurata & emodin & $(91)$ \\
\hline Cladoniaceae & Cladonia spp. & skyrin & $(48)$ \\
\hline \multicolumn{4}{|l|}{ Insects: } \\
\hline Dactylopius & $\begin{array}{l}\text { Dactylopius coccus Costa } \\
\text { (cochineal) }\end{array}$ & carminic acid [food additive E120(ii)] & $(15,22,37,50)$ \\
\hline Porphyrophora & $\begin{array}{l}\text { Porphyrophora hameli } \mathrm{B} \text {. } \\
\& \text { Porphyrophora polonica } \mathrm{L} \text {. }\end{array}$ & carminic acid; flavokermesic acid (LaE); kermesic acid & $(15,44,45)$ \\
\hline Kermes & $\begin{array}{l}\text { Kermes vermilio Planchon } \\
(=\text { Kermococcus vermilio })\end{array}$ & kermesic acid & $(15,44)$ \\
\hline Kerria & Kerria lacca Kerr & laccaic acids (A, B, C, E) & $(15,44,45)$ \\
\hline
\end{tabular}


several known mycotoxins were coproduced in the medium, e.g. secalonic acid D, oxaline, citrinin, tanzawaic acid $\mathrm{A}$, cyclochlorotine, islanditoxin, luteoskyrin, erythroskyrin, rugulosin or aspergiolide A (Table 6). Many of these mycotoxins are pigmented, that is, naphtoquinones from Aspergillus and Penicillium. All these fungal secondary metabolites (on one hand, the yellow and red HAQN pigments that show substitution on both aromatic rings and, on the other hand, the naphtoquinone-type mycotoxins) arise biosynthetically by the same polyketide pathway. The cytotoxic activity of naphtoquinones, and of mycotoxins in general, against mouse leukemia and HeLa cells has been mainly reported in the literature. Moreover, along with the antibiotic and toxic activities, naphtoquinones revealed mutagenic and carcinogenic properties. The results suggested that these fungal strains could not be used to provide safe fungal hydroxyanthraquinoid pigments as potent natural food grade colorants.

Species of Eurotium spp. (E. amstelodami, E. chevalieri and $E$. herbariorum $)^{5,92}$ were found to produce the yellow pigment physcion and the red pigment erythroglaucin (group $A_{1}$ ), however they produce in addition the mycotoxin echinulin and two benzaldehyde coulouring compounds: flavoglaucin (yellow) and auroglaucin (red) (see Table 6). In the same way, it has been demonstrated that a coproduction of red hydroxyanthraquinoid pigments (with no hydroxyl substituents at the positions R1 and R4) and mycotoxins such as fusaric acid, nectriafurone, monoliformin and gibepyrones, occurs by using strains of Fusarium oxysporum isolated from roots of diseased citrus trees ${ }^{95-97}$ (Table 6).

Apart from those mycotoxigenic fungi, there are other filamentous fungi that have the ability to produce known HAQN pigments that arise biosynthetically by the polyketide pathway more particularly, without coproduction of mycotoxins. A strain of Dermocybe sanguinea (= Cortinarius sanguineus) has been identified as producing the red HAQN glycoside dermocybin-1- $\beta$-D-glycopyranoside giving the typical red colour of the fruiting bodies and the spores, in addition with both emodin and physcion pigments ${ }^{3,15,18,52}$. In the fresh fungi as much as $90 \%$ of the pigments exist as glycosides. The detection of emodin-glycosides and physcionglycosides was also pointed from a strain of Dermocytes spp. ${ }^{98}$

Strains of Trichoderma aureoviride ${ }^{99}$ and T. harzianum ${ }^{29,100}$ were found to produce yellow pigment pachybasin (group $\mathrm{E}_{3}$ ) and also the orange-red pigment chrysophanol (group $\mathrm{A}_{1}$ ). Both species of Trichoderma polysporum and T. viride
can also produce pachybasin in addition to the emodin and chrysophanol pigments. Several HAQN-type pigments have been isolated from cultures of Curvularia lunata ${ }^{3-5}$. The main pigments characterized were erythroglaucin (red, group $A_{1}$ ), catenarin (red, group $\mathrm{C}_{1}$ ), chrysophanol (orange-red, group $\mathrm{A}_{1}$ ), helminthosporin (maroon, group $\mathrm{A}_{1}$ ) and cynodontin (bronze, group $\mathrm{A}_{1}$ ). Cynodontin extracted from the biomass of $C$. lunata has been converted successfully to two anthraquinone biodyes (Disperse blue 7 and Acid Green 28). The properties of these biodyes applied to knitted polyamides were compared with those of conventional dyes and found to be identical to all-important aspects ${ }^{3}$. Several species of Drechslera (e.g. D. teres, $D$. graminea, $D$. tritici-repentis, D. phlei, D. dictyoides and $D$. avenae) give $\mathrm{HAQN}$ pigments like catenarin (red, group $\mathrm{C}_{1}$ ), helminthosporin (maroon, group $\mathrm{A}_{1}$ ), cynodontin (bronze, group $A_{1}$ ), tritisporin (reddish brown, group $A_{1}$ ) and erythroglaucin (red, group $A_{1}$ ), without coproduction of mycotoxins ${ }^{3,103}$. Other HAQN pigments like averythrin (orange, group $\mathrm{A}_{1}$ ) and averythrin-6-monomethyl ether were isolated and identified from a culture of Herpotrichia rhodosticta without coproduction of known mycotoxins ${ }^{104}$. More recently, a red pigment produced by a strain of Isaria farinosa was recently elucidated as a chromophore of the anthraquinone type ${ }^{105}$. Similarly, the red pigment produced by Paecilomyces sinclairii, which was beforehand discovered but uncharacterized $^{106}$, is certainly of an identical chemical nature, i.e. an amino group linked to an anthraquinone structure ${ }^{105}$.

\section{Toxicity and carcinogenicity of some natural hydroxyan- thraquinoid pigments}

Anthraquinoid derivatives, including natural HAQN pigments, possess a broad spectrum of biological activities, including anti-inflammatory, anti-cancer, anti-viral, antifungal, anti-bacterial, astringent and purgative. In general, natural HAQN pigments and their intermediates have not been reported as strongly toxic substances, even if it is known that some anthraquinoid dyes are toxic or mutagenic ${ }^{107,108}$. Due to its use as a food colorant in Japan, the safety of European madder extracts has been studied in the literature. For example, in an extensive study the European madder roots were extracted using different solvents and extracts were fractionated by chromatography. Several colour components extracted from madder roots were positive to mutagenicity tests as the yellow rubiadin pigment (group $\mathrm{E}_{3}$; see Table 3) and the red lucidin pigment (group $\mathrm{E}_{3}$ ) aglycones, which are metabolites of lucidin-3-O-primeveroside. From structure mutagenicity studies it was concluded that 1,3-dihydroxyanthraquinones that bear a methyl $\left(-\mathrm{CH}_{3}\right)$ or hydroxymethyl ($\left.\mathrm{CH}_{2} \mathrm{OH}\right)$ group in position $\mathrm{R} 2$, e.g. rubiadin or lucidin, respectively, are mutagenic. For direct mutagenicity an oxygenated state of the benzylic carbon-2 is required. Mutagenic studies about lucidin more particularly showed that a reactive compound is formed from the metabolism of the pigment, which then reacts with DNA and possibly other macromolecules to form covalent adducts ${ }^{109,110}$. Other 1,3dihydroxyanthraquinones that do not possess a methyl or hydroxymethyl group in position R2, such as the orange pigment nordamnacanthal (group $\mathrm{E}_{3}$ ) and the orange-red munjistin pigment (group $\mathrm{E}_{3}$ ), are not found to be mutagenic, since the dehydration to the exomethylenic compound is not possible under physiological conditions ${ }^{15}$. In a 13-week repeated oral dose toxicity study of madder colour, which was performed using F344 rats, the animals were fed a diet containing $0,0.6,1.2,2.5$ or $5.0 \%$ of colouring compounds extracted from madder roots. The results suggested that madder colour exerts mild toxicity, targeting liver, kidneys and possibly red blood cells and white blood cells, some renal changes being evident from $0.6 \%$ madder colour in diet. This is considered to be the lowest-observed adverse effect level $\left(305.8-309.2 \mathrm{mg} / \mathrm{kg}\right.$ of body weight per day) ${ }^{111}$. Data are in agreement with another study performed in the same year in a medium-term multiorgan carcinogenesis bioassay in male F344 rats, which reported that madder colour demonstrated significant tumour-promoting effects in the liver and kidneys ${ }^{112}$. More recently, an additional two-year carcinogenicity study conducted on male and female F344

\section{算 Springer}




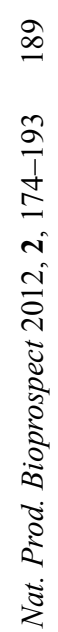
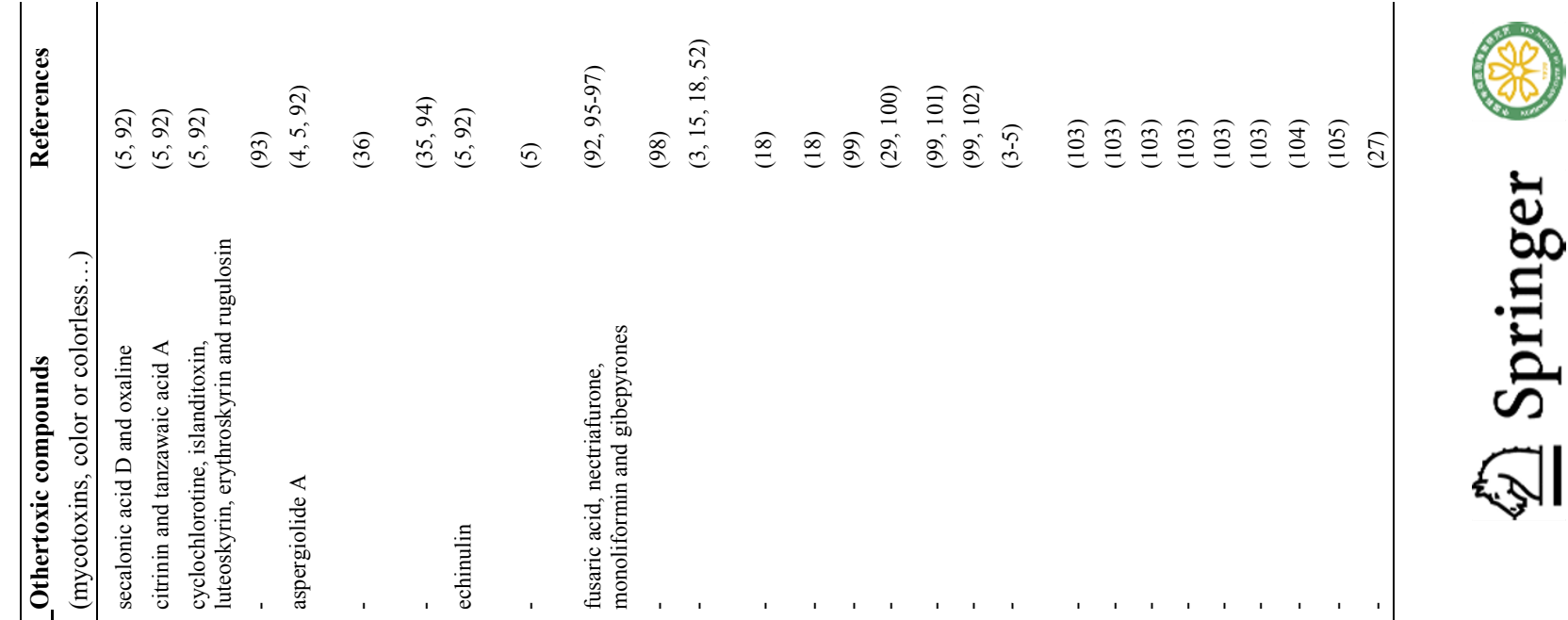

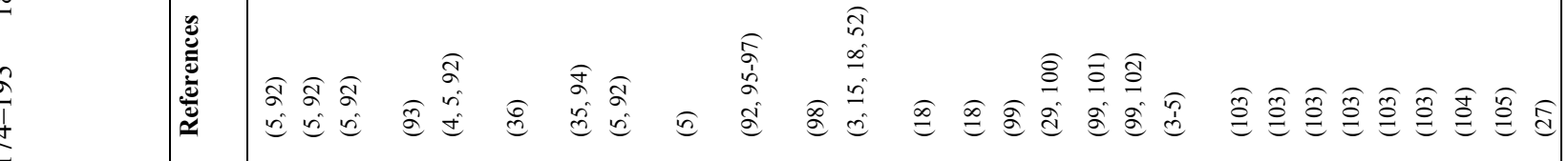
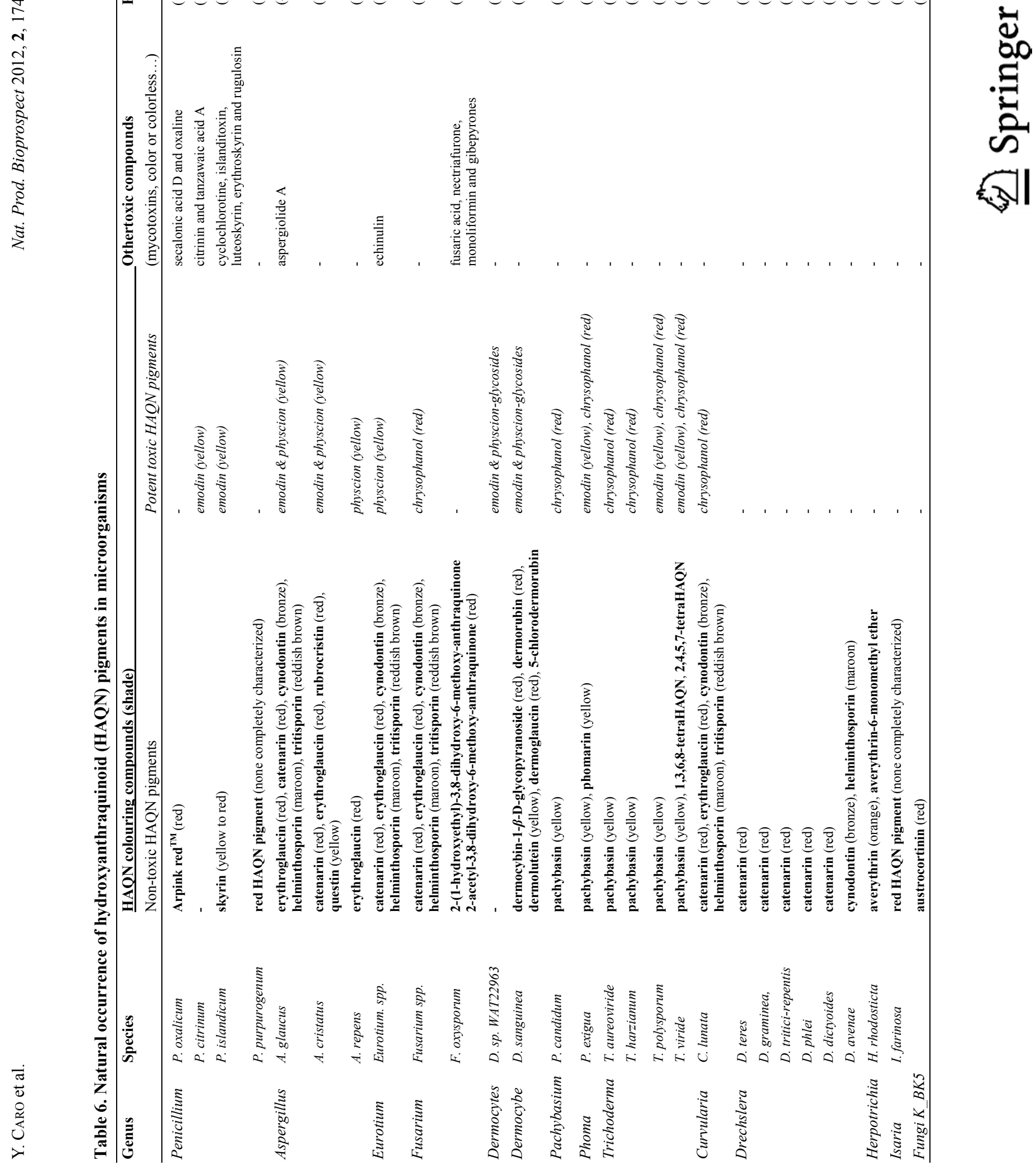
which were fed a diet containing $0,2.5$ or $5.0 \%$ of colouring compounds extracted from madder roots clearly indicate that this dyestuff - rich in alizarin, lucidin-3-O-primeveroside and ruberythric acid, all classified in the 'group $\mathrm{E}_{3}$ ' of HAQN and synthesized via the chorismate/o-succinylbenzoic acid pathway as mentioned above - exerts a carcinogenic potential in both the kidney and the liver, even with the lower dose of the study ${ }^{113}$. These studies support data in other previous studies ${ }^{114,115}$ and provide clear evidence that madder colour exerts unequivocal carcinogenicity against renal tubule cells and hepatocytes in rats. Therefore, the authors ${ }^{113}$ concluded that further studies on these individual HAQN components should be performed to clarify which anthraquinone is responsible for carcinogenicity.

Other recent studies indicate that the dark red purpurin pigment extracted from Indian madder (Rubia cordifolia) classified in the 'group $\mathrm{E}_{3}$ ' of HAQN dyes and synthesized via the chorismate/o-succinylbenzoic acid pathway-has an antimutagenic effect on the Ames Salmonella bacterial mutagenicity assay. The antigenotoxic effect was observed in Drosophila melanogaster against a range of environmental carcinogens. Inhibition of the formation of hepatic DNA adducts in male C57b16 mice after a single dose of the heterocyclic amine dietary carcinogen Trp-P-2 (30 mg/kg) was observed by short-term dietary supplementation with purpurin $^{116}$. In another study, purpurin was found to show inhibition of mutagenicity of a number of heterocyclic amines in the Ames mutagenicity test. The inhibition effect of purpurin was dependent upon $\mathrm{pH}$, being better in neutral than acidic conditions ${ }^{15}$.

Concerning anthraquinoid pigments synthesized via the polyketide pathway, the orange pigment aloe-emodin (group $\mathrm{A}_{1}$ ) induced micronucleus frequencies in the in vitro micronucleus test in mouse lymphoma L5178Y cells ${ }^{117}$. The emodin pigment (group $A_{1}$ ) has toxic and gene mutagenic properties. The activation mechanism of emodin into a direct mutagen to Salmonella typhimurium TA1537 was investigated by using the S9 and microsomes of rat livers. Emodin exhibited mutagenicity in the presence of NADPH or NADH ${ }^{118}$. Another study mentioned that emodin was clearly genotoxic in mouse lymphoma cells ${ }^{119}$. Emodin of fungal origin has been classified as diarrheagenic and genotoxic mycotoxin ${ }^{58}$. Similarly, as fungal chrysophanol and physcion are hypothesized to exert genotoxicity, they are also considered as mycotoxins today ${ }^{92}$. Thus the detection of emodin, physcion and/or chrysophanol from some strains of Aspergillus spp., Penicillium spp., Eurotium spp., Dermocybe sanguinea, Dermocytes spp., Trichoderma spp. and Curvularia lunata (see Table 6) suggests that some of these fungi are potent mycotoxigenic.

\section{Current industrial applications of natural hydroxyan- thraquinoid dyestuffs}

Traditionally, relevant hydroxyanthraquinoid dyestuffs such as the famous kermes parasite insect, the cochineal insect and the European madder root are essentially used to dye textiles. They are also used for other non-food applications, e.g. printing, cosmetics (hair colorants...) and pharmaceutical applications across the globe. They provide the most important red pigments used in artistic paintings. Hydroxyanthraquinoid red dyes were among the reds that dominated the dye markets of Europe. Natural hydroxyanthraquinoid pigments were generally applied as isolated compounds or as glycosides. It was discovered that natural hydroxyanthraquinoid pigments, like alizarin from madder root and carminic acid from cochineal insect, coloured hair directly even at room temperature and that they were resistant to perspiration, washing, light and adverse weather conditions. Furthermore, hydroxyanthraquinoid pigments were very stable in solutions of the cosmetic media ${ }^{15}$.

Colour compounds extracted from the roots of European madder have been used as mentioned above in Japan as colorants for food, e.g. confectionery, boiled fish and soft drinks, but they are not allowed as a food additive in either the US or the EU. Only the natural red colorant 'cochineal extract' (additive E-120(ii)) which is an extract of the dried bodies of the female cochineal insect, with around $20 \%$ carminic acid content, is allowed and widely used as a colouring agent in food processes in the EU (at dosage levels from 50 to 500 $\mathrm{mg} / \mathrm{kg}$ ) and in the US (only up to $5 \mathrm{mg} / \mathrm{kg}$ ) ${ }^{7,15,22,38}$. Cochineal is commonly cultivated from the wild prickly pear cactus that grows thickly on the mountainsides in central Peru. Cochineal produces the pigment as a deterrent against other insects. The pigment can be obtained from the body and eggs of the insect. The few countries that produce commercial cochineal extracts are Peru, Mexico, the Canary Islands and, more recently, Chile and Bolivia. Only in Peru, the commercial production of cochineal extract is 200 ton/year, whereas in the Canary Islands production is only about 20 ton/year ${ }^{7}$. France is believed to be the world's largest importer of cochineal extract, but Italy and Japan come next. The insects are killed by immersion in hot water or hot ethanol or by exposure to sunlight, steam, or by oven heat. Approximately 130,000 insects or $2 \mathrm{~kg}$ dry insects are required to produce $1 \mathrm{~kg}$ of cochineal extract and approximately $200 \mathrm{~kg}$ of dried insects are produced weekly at the largest cochineal farm ${ }^{7}$. Cochineal extract is very soluble in water and exhibits shade changes with changes in $\mathrm{pH}$. At $\mathrm{pH} 4$ and below, it is orange; it turns from violet to red by increasing $\mathrm{pH}$ from 5 to 7 . Traditionally, cochineal extract is extracted with water or aqueous alcohol at $90{ }^{\circ} \mathrm{C}$ to $100{ }^{\circ} \mathrm{C}$ by batch or continuous process ${ }^{7}$. It is one of the few natural and water-soluble colorants that resist degradation with time. It has a good stability to heat, chemical oxidation, light and oxygen ${ }^{15}$. Often it is more stable than some synthetic food grade colorants but instable at low $\mathrm{pH}$. A water insoluble form of cochineal extract is commonly used to colour several food products, e.g. sausage products, bakery and dairy products, confectionery, and often competes with red root beet (betanin) and anthocyanins in food colouring. The water-soluble form of cochineal extract is currently used in beverages, soft and alcoholic drinks such as aperitifs (e.g. Campari $\left.^{\mathrm{TM}}\right)$. Cochineal extract is not kosher and is not vegetarian. Its main limitation in food application is it's insolubility at low $\mathrm{pH}$ as mentioned above ${ }^{15}$. Carmine, i.e. the additive E-120(i), is a complex of carminic acid with various metals: an aluminium lake of carminic acid is currently being used in the commercial preparation of carmine $e^{7,15,22}$. Variation in the ratio of carminic acid to aluminium produces a range of colours from pale strawberry to near black currant. Carmine is commonly traded as powder with a carminic acid content of $40 \%$ to $60 \%$ and liquid aqueous alkaline forms of carmine (and spray-dried derivatives) are also available with a carminic acid content of $2 \%$ to $7 \%$. Cochineal extract and carmine are neither toxic nor known to be carcinogenic. Carmine is widely consumed in foods and beverages and has been rarely

\section{然 Springer}


implicated in adverse reactions. It can induce an anaphylacticshock reaction in a small number of people, due to impurities in the preparation, not due to the pigment itself. In fact, colouring compounds in natural food grade colorants are small molecular weight, non-protein chemicals that cannot be expected to give true food allergies, either immunoglobulin $\mathrm{E}$ (IgE)-mediated or cell-mediated allergy. However, natural colorants, for example carmine, are often extracted from biological materials that may contain many other compounds, including proteins in addition to the colouring compounds. In 1998, it was reported that IgE-mediated allergy might be caused by the consumption of carmine, due to the presence of protein residues ${ }^{120}$. Once IgE sensitization to these carmine proteins occurs, the level of exposure to these residual proteins through carmine-containing foods and beverages may be sufficient to elicit allergic reactions. For example, an anaphylactic reaction has been reported in a 34-year-old female atopic patient after ingestion of an orange beverage containing carmine. Symptoms like urticaria, rhinitis, nausea, vomiting, asthma, chills and diarrhea were observed. Skin prick tests carried out on the orange beverage, carmine and cosmetics containing the pigment were positive. In 1995, a reaction to carmine occurred in a 35-year-old woman after she ingested yoghurt that contained mixed fruits. Approximately 2 $\mathrm{h}$ after consumption she experienced symptoms of anaphylaxis including generalized urticaria, angioedema (localized swelling) and asthma ${ }^{121}$. In 1997, four adverse reactions following consumption of an alcoholic beverage containing carmine were reported in women ranging from 25 to 43 years old, with urticaria and angioedema. A skin prick test was performed and was found positive for carmine contained in the alcoholic beverage. Four instances of acute allergic reactions in a 28-year-old female after ingestion of orange beverage, strawberry milk and a red coloured cocktail containing carmine were also mentioned. An anaphylactic reaction in a 27-year-old woman has been reported after the consumption of a Popsicle coloured with carmine ${ }^{122}$. Carmine and cochineal extract are different from the azo pigment 'cochineal red A' (additive E-124) which is a synthetic colorant.

Concerning the natural food colorant Arpink red ${ }^{\mathrm{TM}}$, many toxicological data are also available: acute oral toxicity in mice of the pigment, 90-day subchronical toxicological study, acute dermal irritation/corrosion, acute eye irritation/corrosion, anti-tumour effectiveness, micronucleus test in mice, AMES test (Salmonella typhimurium reverse mutation assay), estimation of antibiotic activity, results of estimation of five mycotoxins. The fungal colorant gives a raspberry-red colour in an aqueous solution, stable at $\mathrm{pH}$ over 3.5. Neutral solutions are stable even after 30 min of boiling and colour shade does not change in relation with $\mathrm{pH}^{2}$. After evaluating all the materials provided by the Ascolor Biotech s.r.o company, the Codex Alimentarius Commission (Rotterdam meeting, March 11-15, 2002) made the following statement: "there will not be any objections to use the red colouring matter Arpink red ${ }^{\mathrm{TM}}$ "; The Arpink red $^{\mathrm{TM}}$ colorant use was recommended as 100 $\mathrm{mg} / \mathrm{kg}$ in meat products and in non-alcoholic drinks, 200 $\mathrm{mg} / \mathrm{kg}$ in alcoholic drinks, $150 \mathrm{mg} / \mathrm{kg}$ in milk products including ice creams and $300 \mathrm{mg} / \mathrm{kg}$ in confectionery products $^{2}$. After the first approval by the Codex Alimentarius, the Arpink red ${ }^{\mathrm{TM}}$ safety assessment was discussed during the 63rd meeting of Joint FAO/WHO Expert Committee on Food Additives (JECFA) in Geneva, June 8-17, 2004. The red colorant received a two-year temporary approval by the EU for distribution as a food additive, exclusively in the Czech Republic from 2004 to $2006^{2}$. The file was still under progress at the European Food Safety Authority (EFSA) for some years. The situation now is not clear as Ascolor Biotech s.r.o. did not send data to authorities later on and seems to have closed its activities. Thus, there is no particular information on potential mycotoxin production and pathogenicity towards humans, despite the fact that the production of secalonic acid $\mathrm{D}$, i.e. a pale yellow teratogenic mycotoxin, is well known from the fungus Penicillium oxalicum ${ }^{5}$. It has been shown that the biosynthesis of secalonic acid D (see Fig. 2 for the chemical structure) was dependent on the biosynthesis of the pigment emodin via the acetate-malonate pathway in a study conducted on the lichen Laurera benguelensis ${ }^{41}$. Diverse biological activities of secalonic acid D have been reported, such as a mycotoxin towards chicken and mice embryo, an inhibitor of various isozymes of protein kinase $\mathrm{C}$ and protein kinase $\mathrm{A}$ in murine secondary palate development ${ }^{123}$, as well as mouse and human cleft palatal inducing agent ${ }^{124}$.

In conclusion, this review provides relevant information regarding the properties of hydroxyanthraquinoid pigments, their biosynthetic pathway, their toxicity and carcinogenicity in recent decades. The collective information summarized in the review will act as an important segment for development of 'niche' fungal dyestuffs rich in hydroxyanthraquinoid pigments. These conclusions indicate that, even if the toxicological investigations of a new additive are not financially negligible, non-mycotoxigenic filamentous fungi such as strains of Drechslera spp., Herpotrichia spp., Paecilomyces spp. and Isaria spp. could be used for the production of dyestuffs rich in hydroxyanthraquinoid pigments as potent natural food grade colorants, with different shades according to the biomass composition: such as red (for main components catenarin \& erythroglaucin), reddish brown (for tritisporin), bronze (for cynodontin), maroon (for helminthosporin) and orange-yellow (for pachybasin \& averythrin). However, further studies should be performed on these fungal HAQN pigments to evaluate their potent carcinogenicity in humans from the food safety perspective. Current data on this topic are therefore insufficient.

\section{Acknowledgments}

The authors are grateful to Valérie QUILICI and Lynda GOYER from Université de La Réunion who helped in the preparation of this review.

Open Access This article is distributed under the terms of the Creative Commons Attribution License which permits any use, distribution, and reproduction in any medium, provided the original author(s) and source are credited.

\section{References}

[1] Downham, A.; Collins, P. Int. J. Food Sci. Technol. 2000, 35, 522.

[2] Dufossé, L. Food Technol. Biotech. 2006, 44, 313-321.

[3] Räisänen, R. Handbook of Natural Colorants; John Wiley \& Sons: Chichester, 2009; Chapter 11, pp 183-200.

[4] Duran, N.; Maria, F. S. T.; Roseli, D. C.; Elisa, E. Crit. Rev. Food. Sci. Nutr. 2002, 42, 53-66.

[5] Mapari, S. A. S.; Nielsen, K. F.; Larsen, T. O.; Frisvad, J. C.; Meyer, A. S.; Thrane, U. Curr. Opin. Biotechnol. 2005, 16, 231- 
238

[6] Dufossé, L.; Galaup, P.; Yaron, A.; Arad, S. M.; Blanc, P.; Chidambara-Murthy, K. N.; Ravishankar, G. A. Trends Food Sci. Tech. 2005, 16, 389-406.

[7] Chattopadhyay, P.; Chatterjee, S.; Sen, S. K. Afr. J. Biotechnol. 2008, 7, 2972-2985.

[8] Mapari, S. A. S.; Meyer, A. S.; Thrane, U.; Frisvad, J. C. Microbial. Cell. Factories. 2009, 8, 24-28.

[9] Blanc, P. J.; Loret, M. O.; Santerre, A. L.; Pareilleux, A.; Promé, D.; Promé, J. C. J. Food Sci. 1995, 59, 862-865.

[10] Hajjaj, H.; Blanc, P. J.; Groussac, E.; Goma, G.; Uribelarrea, J. L.; Loubiere, P. Enzyme Microb. Technol. 2000, 27, 619-625.

[11] Carvalho, J. C.; Pandey, A.; Batitha, S.; Soccol, C. R. Agro Food Ind Hi-tech. 2003, 14, 37-42.

[12] Liu, B. H.; Wu, T. S.; Su, M. C.; Chung, C. P.; Yu, F. Y. J. Agric. Food Chem. 2005, 53, 170-175.

[13] Mapari, S. A. S.; Thrane, U.; Meyer, A. S. Trends Biotechnol. 2010, 28, 300-307.

[14] Liu, R.; Lu, Y.; Wu, T.; Pan, Y. Chromatographia 2008, 68, 9599.

[15] Bechtold, T. Handbook of Natural Colorants; John Wiley \& Sons: Chichester, 2009; Chapter 10, pp 151-182.

[16] Alitheen, N. B.; Mashitoh, A. R.; Yeap, S. K.; Shuhaimi, M.; Abdul-Manaf, A.; Nordin, L. Int. Food Res. J. 2010, 17, 711719 .

[17] Bonose-Crosnier de Bellaistre, M.; Nowik, W.; Tchapla, A.; Heron, S. J. Chromatogr. 2011, 1218, 778-786.

[18] Bick, I. R. C.; Rhee, C. Biochem. J. 1966, 98, 112-116.

[19] De Santis, D.; Moresi, M. Ind. Crop. Prod. 2007, 26, 151-162.

[20] Baker, R. A.; Tatum, J. H. J. Ferment. Bioeng. 1997, 85, 359361.

[21] Nagia, F. A.; El-Mohamedy, R. S. R. Dyes Pigments 2007, 75, $550-555$.

[22] Socaciu C. Food Colorants-Chemical and functional properties; CRC Press Taylor \& Francis: New York, 2008.

[23] Drivas, I.; Blackburn, R. S.; Rayner, C. M. Dyes Pigments 2011, $88,7-17$.

[24] Liu, S. Y.; Sporer, F.; Wink, M.; Jourdane, J.; Henning, R.; Li, Y. L.; Ruppel, A. Trop. Med. Int. Health 1997, 2, 179-188.

[25] Locatelli, M.; Tammaro, F.; Menghini, L.; Carlucci, G.; Epifano, F.; Genovese, S. Phytochem. Lett. 2009, 2, 223-226.

[26] Kremer, D.; Kosalec, I.; Locatelli, M.; Epifano, F.; Genovese, S.; Carlucci, G.; Zovko Koncic, M. Food Chem. 2012, 131, 11741180 .

[27] Muangsin, N.; Wisetsakdakorn, W.; Chaichit, N.; Sihanonth, P.; Petsom, A.; Sangvanich, P. Dyes Pigments 2008, 77, 653-656.

[28] Tanaka, C.; Miyagawa, H.; Kuwahara, Y.; Tsuda, M. Mycoscience 2002, 43, 317-320.

[29] Liu, S. Y.; Lo, C. T.; Chen, C.; Liu, M. Y.; Chen, J. H.; Peng, K. C. J. Biochem. Biophys. Methods 2007, 70, 391-395.

[30] Yadav, J. P.; Arya, V.; Panghal, M.; Kumar, S.; Dhankhar, S. Fitoterapia 2010, 81, 223-230.

[31] Zhang, L. M.; Xie, W. G.; Wen, T. T. J. Therm. Anal. Calorim. 2010, 100, 215-218.

[32] Gill, M.; Steglich, W. Progress in the Chemistry of Organic Natural Products; Springer: New York, 1987; Chapter 51, pp 125-174.

[33] Manojlovic, I.; Bogdanovic-Dusanovic, G.; Gritsanapan, W.; Manojlovic, N. Chem. Pap. 2006, 60, 466-468.

[34] Orban, N.; Boldizsar, I.; Szücs, Z.; Danos, B. Dyes Pigments 2008, 77, 249-257.

[35] Smetanina, O. F.; Kalinovskii, A. I.; Khudyakova, Y. V.; Slinkina, N. N.; Pivkin, M. V.; Kuznetsova, T. A. Chem. Nat. Compd. 2007, 43, 327-329.

[36] Anke, H.; Kolthoum, I.; Zihner, H.; Laatsch, H. Arch. Microbiol. 1980, 126, 223-230.

[37] Ackacha, M. A.; Polec-Pawlak, K.; Jarosz, M. J. Sep. Sci. 2003, $26,1028-1034$.
[38] Banyai, P.; Kuzovkina, I. N.; Kursinszki, L.; Szöke, E. Chromatographia 2006, 63, 111-114.

[39] Jasril; Lajis, N. H.; Mooi, L. Y.; Abdullah, M. A.; Sukari, M. A.; Ali, A. M. Asia Pacific J. Mol. Biol. Biotechnol. 2003, 11, 3-7.

[40] Bao, L.; Liu, L.; Wu, Y.; Han, T.; Xue, L.; Zhang, Q. Chem-Biol. Interact. 2011, 194, 97-105.

[41] Manojlovic, N. T.; Vasiljevic, P. J.; Gritsanapan, W.; Supabphol, R.; Manojlovic, I. Biol. Res. 2010, 43, 169-176.

[42] Tanaka, C.; Miyagawa, H.; Kuwahara, Y.; Tsuda, M. Mycoscience 2002, 43, 317-320.

[43] Tang, T.; Yin, L.; Yang, J.; Shan, G. Eur. J. Pharmacol. 2007, $567,177-185$.

[44] Schweppe, H. Historic Textile and Paper Materials II; American Chemical Society: Washington, 1989; Chapter 13, pp 188-219.

[45] Rafaëlly, L.; Héron, S.; Nowik, W.; Tchapla, A. Dyes Pigments 2008, 77, 191-203

[46] Mishchenko, N. P.; Stepanenko, L. S.; Krivoshchekova, O. E.; Maksimov, O. B. Chem. Nat. Compd. 1980, 16, 117-121.

[47] Borroto, J.; Coll, J.; Rivas, M.; Blanco, M.; Concepcion, O. Tandron, Y. A.; Hernandez, M.; Trujillo, R. Plant Cell Tiss. Org. 2008, 94, 181-187

[48] Yamamoto, Y.; Matsubara, H.; Kinoshita, Y.; Kinoshita, K.; Koyama, K.; Takahashi, K.; Ahmadjam, V.; Kurokawa, T.; Yoshimura, I. Phytochemistry 1996, 43, 1239-1242.

[49] Gagunashvili, A. N.; Davidsson, S. P.; Jonsson, Z. O.; Andresson, O. S. Mycol. Res. 2009, 113, 354-363.

[50] Canamares, M. V.; Garcia-Ramos, J. V.; Domingo, C.; SanchezCortes, S. Vib. Spectrosc. 2006, 40, 161-167.

[51] Bouras, N.; Strelkov, S. E. Physiol. Mol. Plant P. 2008, 72, 8795 .

[52] Birkinshaw, J. H.; Gourlay, R. Biochem. J. 1961, 80, 387-392.

[53] Miliani, C.; Romani, A.; Favaro, G. J. Phys. Org. Chem. 2000, $13,141-150$.

[54] Van den Berg, A. J. J.; Labadie, R. P. Methods in Plant Biochemistry; Academic press: London, 1989; Vol. 1, pp 451-491

[55] Bringmann, G.; Gulder, T. A. M.; Hamm, A.; Goodfellow, M.; Fiedler, H. P. Chem. Commun. 2009, 44, 6810-6812.

[56] Leistner, E. Primary and secondary metabolism of plant cell cultures; Springer: Berlin, 1985; pp 215-224

[57] Han, Y. S.; Van der Heijden, R.; Lefeber, A. W. M.; Erkelens, C.; Verpoorte, R. Phytochemistry 2002, 59, 45-55.

[58] Izhazi, I. New Phytol. 2002, 155, 205-217.

[59] Angelini, L. G.; Pistelli, L.; Belloni, P.; Bertoli, A.; Panconesi, S. Ind. Crop. Prod. 1997, 6, 303-311.

[60] Kaur, P.; Chandel, M.; Kumar, S.; Kumar, N.; Singh, B.; Kaur, S. Food Chem. Toxicol. 2010, 48, 320-325.

[61] Morimoto, M.; Tanimoto, K.; Sakatani, A.; Komai, K. Phytochemistry 2002, 60, 163-166.

[62] El-Gamal, A. A.; Takeya, K.; Itokawa, H.; Halim, A. F.; Amer, M. M.; Saad, H. E. A.; Awad, S. A. Phytochemistry 1995, 40, 245-251

[63] Banthorpe, D. V.; White, J. J. Phytochemistry 1995, 38, 107-111.

[64] Koyama, J.; Ogura, T.; Tagahara, K. Phytochemistry 1993, 33, 6, $1540-1542$

[65] Sang, S.; Cheng, X.; Zhu, N.; Stark, R. E.; Badmaev, V.; Ghai, G.; Rosen, R. T.; Ho, C. T. J. Agric. Food Chem. 2001, 49 , 4478-4481.

[66] Wijnsma, R.; Verpoorte, R.; Mulder-Krieger, T.; Baerheim Svendsen, A. Phytochemistry 1984, 23, 2307-2311.

[67] Robins, R. I.; Payne, J.; Rhodes, M. J. C. Phytochemistry 1986, $25,2327-2334$

[68] Wijnsma, R.; Go, J. T. K. A.; Harkes, P. A. A.; Verpoorte, R.; Baerheim Svendsen, A. Phytochemistry 1986, 25, 1123-1126.

[69] Khouri, H. E.; Ibrahim, R. K. Phytochemistry 1987, 26, 25312535

[70] Schripsema, J.; Ramos-Valdivia, A.; Verpoorte, R. Phytochemistry 1999, 51, 55-60.

[71] Arun, P.; Purushotham, K. G.; Jonhsy Jayarani, J.; Vasantha Kumari. J. Pharm. Sci. Technol. 2010, 2, 198-201. 
[72] Siva, R.; Mayes, S.; Behera, S. K.; Rajasekaran, C. Ind. Crop. Prod. 2012, 37, 415-419.

[73] Badr, J. M. Nat. Prod. Sci. 2008, 14, 227-232.

[74] Liu, B.; Ge, X.; He, Y.; Xie, J.; Xu, P; He, Y.; Zhou, Q.; Pan, L.; Chen, R. Aquaculture 2010, 310, 13-19.

[75] Li, L.; Zhang, C.; Xiao Y. Q.; Chen, D. D.; Tian, G. F.; Wang, Y. Chin. J. Nat. Med. 2011, 9, 410-413.

[76] Malik, S.; Sharma, N.; Sharma, U. K.; Singh, N. P.; Blushan, S.; Sharma, M.; Sinha, A. K.; Ahuja, P. S. J. Plant Physiol. 2010, 167, 749-756.

[77] Baskan, S.; Daut-Özdemir, A.; Günaydin, K.; Erim, F. B. Talanta 2007, 71, 747-750.

[78] Choi, G. J.; Lee, S. W.; Jang, K. S.; Kim, J. S.; Cho, K. Y.; Kim, J. C. Crop Prot. 2004, 23, 1215-1221.

[79] Lee, N. J.; Choi, J. H.; Koo, B. S.; Ryu, S. Y.; Han, Y. H.; Lee, S. I.; Lee, D. U. Biol. Pharm. Bull. 2005, 28, 2158-2161.

[80] Fairbairn, J. W.; El-Muhtadi, F. J. Phytochemistry 1972, 11, 263-268.

[81] Genovese, S.; Tammaro, F.; Menghini, L.; Carlucci, G.; Epifano, F.; Locatelli, M. Phytochem. Anal. 2010, 21, 261-267.

[82] Panichayupakaranant, P.; Sakunpak, A.; Sakunphueak, A. J. Chromatogr. Sci. 2009, 47, 197-200.

[83] Dagne, E.; Yenesew, A.; Asmellash, S.; Demissew, S.; Mavi, S. Phytochemistry 1994, 35, 401-406.

[84] Van Wyk, B. E.; Yenesew, A.; Dagne, E. Biochem. Syst. Ecol. 1995, 23, 267-275.

[85] Mohanlall, V.; Steenkamp, P.; Odhav, B. J. Med. Plants Res. 2011, 5, 3132-3141.

[86] Rosso, M. L.; Bertoni, M. D.; Adler, M. T.; Maier, M. S. Biochem. Syst. Ecol. 2003, 31, 1197-1200.

[87] Manojlovic, N.; Markovic, Z.; Gritsanapan, W.; Boonpragob, K. Russ. J. Phys. Chem. 2009, 83, 1554-1557.

[88] Piatelli, M.; Giudici de Nicola, M. Phytochemistry 1988, 7, 1183-1187.

[89] Nakano, H.; Komiya, T.; Shibata, S. Phytochemistry 1972, 11, 3505-3508.

[90] Sochting, U.; Fröden, P. Mycol. Prog. 2002, 1, 257-266.

[91] Cohen, P. A.; Neil Towers, G. H. Phytochemistry 1996, 42, 1325-1329.

[92] Frisvad, J. C. Arch. Environ. Contam. Toxicol. 1989, 18, 452467.

[93] Mendez, A.; Perez, C.; Montanez, J.; Martinez, G.; Aguilar, C. N. J. Zhejiang Univ-Sc. B 2011, 12, 961-968.

[94] Podojil, M.; Sedmera, P.; Vokocun, J.; Betina, V.; Barathov, H.; Durackov, K.; Horakova, K.; Nemec, P. Folia Microbiol. 1979, $23,438-443$

[95] Baker, R. A.; Tatum, J. H. J. Ferment. Bioeng. 1998, 85, 359361.

[96] Medentsev, A. G.; Akimenko, V. K. Phytochemistry 1998, 47, 935-959.

[97] Nagia, F. A.; El-Mohamedy, R. S. R. Dyes Pigments 2007, 75, $550-555$.

[98] Gill, M.; Morgan, P. M. ARKIVOC 2001, 145-156.

[99] Reino, J. L.; Guerrero, R. F. Phytochem. Rev. 2008, 7, 89-123.
[100] Vinale, F.; Marra, R.; Scala, F.; Ghisalberti, E. L.; Lorito, M.; Sivasithamparam, K. Lett. Appl. Microbiol. 2006, 43, 143-148.

[101] Donnelly, D. M. X.; Helen, M. S. Phytochemistry 1986, 25 2303-2304.

[102] Slater, G. P.; Haskins, R. H.; Hogge, L. R.; Nesbitt, L. R. Can. J. Chem. 1967, 45, 92-96.

[103] Engström, K.; Brishammar, S.; Svensson, C.; Bengtsson, M.; Andersson, R. Mycol. Res. 1993, 97, 381-384.

[104] Van Eljk, G. W.; Roeijmans, H. J. Exp. Mycol. 1984, 8, 266268

[105] Cho, Y. J.; Park, J. P.; Hwang, H. J.; Kim, S. W.; Choi, J. W. J. Biotechnol. 2002, 95, 13-23.

[106] Velmurugan, P.; Lee, Y. H.; Nanthakumar, K.; Kamala-Kannan, S.; Dufossé, L.; Mapari, S. A. S.; Oh, B. T. J. Basic Microbiol. 2010, 50, 1-10.

[107] Wang, Y.; Chen, J. W.; Ge, L. K.; Wang, D. G.; Cai, X. Y.; Huang, L. P.; Hao, C. Dyes Pigments 2009, 83, 276-280.

[108] Barbee, G. C.; Santer, M. M.; McClain, W. R. Crop Prot. 2010, 29, 506-508.

[109] Kawasaki, Y.; Goda, Y.; Yoshihira, K. Chem. Pharm. Bull. (Tokyo) 1992, 40, 1504-1509.

[110] Marec, F.; Kollarova, I.; Jegorov, A. Planta Med. 2001, 67, $127-131$.

[111] Inoue, K.; Shibutani, M.; Masutomi, N.; Toyoda, K.; Takagi, H.; Uneyama, C.; Nishikawa, A.; Hirose, M. Food Chem. Toxicol. 2008, 46, 241-252.

[112] Yokohira, M.; Yamakawa, K.; Hosokawa, K.; Matsuda, Y.; Kuno, T.; Saoo, K.; Imaida, K. J. Food Sci. 2008, 73, T26-T32.

[113] Kaoru, I.; Midori, Y.; Miwa, T.; Makoto, S.; Hironori, T.; Masao, H.; Akiyoshi, N. Food Chem. Toxicol. 2009, 47, 184 191

[114] Westendorf, J.; Marquardt, H.; Poginsky, B.; Dominiak, M.; Schmidt, J.; Marquardt, H. Mutat Res. 1990, 240, 1-12.

[115] Westendorf, J.; Pfau, W.; Schulte, A. Carcinogenesis 1998, 19, 2163-2168.

[116] Marczylo, T.; Sugiyama, C.; Hayatsu, H. J. Agric. Food Chem. 2003, 51, 3334-3337.

[117] Mueller, S. O.; Stopper, H.; Dekant, W. Drug Metab. Dispos. 1998, 26, 540-546.

[118] Masuda, T.; Ueno, Y. Mutat Res. 1984, 125, 135-144.

[119] Mueller, S. O.; Lutz, W. K.; Stopper, H. Mutat. Res. 1998, 414, $125-129$.

[120] Acero, S.; Tabar, A. I.; Alvarez, M. J.; Garcia, B. E.; Olaguibel, J. M.; Moneo, I. Allergy 1998, 53, 897-901.

[121] Beaudouin, E.; Kanny, G.; Lambert, H.; Fremont, S.; MoneretVautrin, D. Ann. Allergy Asthma Immunol. 1995, 74, 427-430.

[122] Baldwin, J. L.; Chou, A. H.; Solomon, W. R. Ann. Allergy Asthma Immunol. 1997, 79, 415-419.

[123] Hanumegowda, U. M.; Judy, B. M.; Welshons, W. V.; Reddy, C. S. Toxicol. Sci. 2002, 66, 159-165.

[124] Dhulipala, V. C.; Maddali, K. K.; Welshons, W. V.; Reddy, C. S. Birth Defects Res. B Dev. Reprod. Toxicol. 2005, 74, 233 242 .

\section{盟 Springer}

